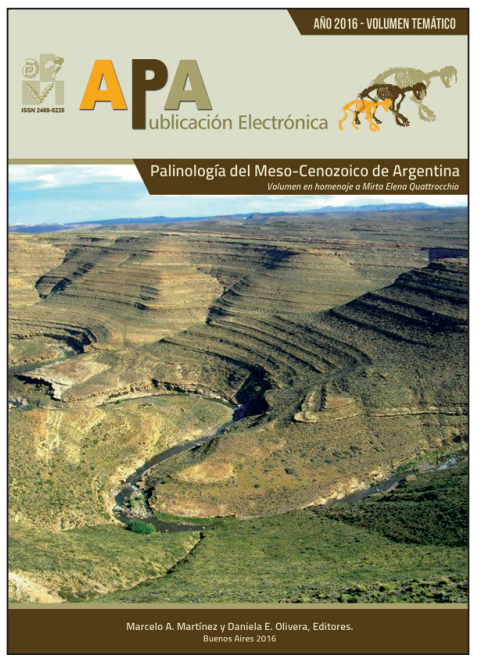

ANÁLISIS E INTERPRETACIÓN DE REGISTROS PALINOLÓGICOS DE SUCESIONES ALUVIALES DE LA REGIÓN PAMPEANA: REVISIÓN E IMPLICANCIAS PARA LA RECONSTRUCCIÓN DE LA VEGETACIÓN Y EL CLIMA DURANTE EL PLEISTOCENO TARDÍO-HOLOCENO

ALDO R. PRIETO

Instituto de Investigaciones Marinas y Costeras, CONICET-Universidad Nacional de Mar del Plata, Laboratorio de Paleoecología y Palinología, Funes 3250,7600 Mar del Plata, Argentina.

Recibido: 29 de abril de 2016 - Aceptado: 27 de septiembre de 2016

Para citar este artículo: Aldo R. Prieto (2016). Análisis e interpretación de registros palinológicos de sucesiones aluviales de la región pampeana: revisión e implicancias para la reconstrucción de la vegetación y el clima durante el Pleistoceno Tardío-Holoceno. En: M. Martínez y D. Olivera (Eds.), Palinología del Meso-Cenozoico de Argentina - Volumen en homenaje a Mirta Elena Quattrocchio. Publicación Electrónica de la Asociación Paleontológica Argentina 16 (2): 148-167.

Link a este artículo: http://dx.doi.org/10.5710/PEAPA.27.09.2016.114

DESPLAZARSE HACIA ABAJO PARA ACCEDER AL ARTÍCULO

Asociación Paleontológica Argentina Maipú $6451^{\circ}$ piso, C1006ACG, Buenos Aires República Argentina Tel/Fax (54-11) 4326-7563 Web: www.apaleontologica.org.ar

Otros artículos en Publicación Electrónica de la APA 16(2):

\section{Guler, Paolillo \& Martz}

EARLY CRETACEOUS

DINOFLAGELLATE CYSTS FROM

THE NEUQUÉN AND AUSTRAL

BASINS: A REVIEW

\section{Deschamps \& Tomassini}

LATE CENOZOIC VERTEBRATES FROM

THE SOUTHERN PAMPEAN REGION:

SYSTEMATIC AND

BIO-CHRONOSTRATIGRAPHIC UPDATE

\section{Candel \& Borromei}

REVIEW OF THE PALAEOENVIRONMENTAL RECONSTRUCTION OF LATE QUATERNARY MARINE SEQUENCES, TIERRA DEL FUEGO (ARGENTINA) 


\title{
ANÁLISIS E INTERPRETACIÓN DE REGISTROS PALINOLÓGICOS DE SUCESIONES ALUVIALES DE LA REGIÓN PAMPEANA: REVISIÓN E IMPLICANCIAS PARA LA RECONSTRUCCIÓN DE LA VEGETACIÓN Y EL CLIMA DURANTE EL PLEISTOCENO TARDÍO-HOLOCENO
}

\author{
ALDO R. PRIETO
}

Instituto de Investigaciones Marinas y Costeras, CONICET-Universidad Nacional de Mar del Plata, Laboratorio de Paleoecología y Palinología, Funes 3250,7600 Mar del Plata, Argentina. aprieto@mdp.edu.ar

\begin{abstract}
Resumen. En este trabajo se sintetizan y discuten los resultados de ca. 30 años de estudios palinológicos de depósitos aluviales del Pleistoceno Tardío-Holoceno de la región pampeana (RP). La mayor parte de las reconstrucciones de la vegetación para ese lapso han derivado del análisis polínico de depósitos aluviales, aunque en ciertos casos los resultados son controvertidos. La presente revisión muestra que el análisis palinológico de sucesiones aluviales es un recurso importante de información paleoambiental cuali y cuantitativa para la RP, pero también expone que no todas las secuencias polínicas publicadas son adecuadas para hacer reconstrucciones de la vegetación y el clima. Las principales causas de discrepancias entre los resultados han sido el escaso entendimiento sobre el transporte fluvial del polen, la preservación polínica y la tafonomía, y el control de las facies sedimentarias en las posibles modificaciones de los registros polínicos. Las causas de la alteración de los conjuntos polínicos, aunque relacionadas principalmente con repetidos ciclos de hidratación-desecación, degradación microbiana y posible control de facies, han sido escasamente discutidas en la mayoría de los trabajos. Esto señala la necesidad de realizar estudios sobre la preservación polínica diferencial y la cuantificación del polen transportado por los ríos, poniendo más atención en el conocimiento de los procesos tafonómicos para mejorar la interpretación de los registros palinológicos fósiles. A pesar de esto, los progresos realizados recientemente han permitido lograr reconstrucciones significativamente más precisas e informativas de la vegetación y del clima del pasado en la RP que lo que fue posible anteriormente.
\end{abstract}

Palabras clave. Palinología fluvial. Tafonomía. Preservación polínica. Argentina.

Abstract. ANALYSES AND INTERPRETATION OF PALYNOLOGICAL RECORDS FROM ALLUVIAL SEQUENCES OF THE PAMPAS REGION: REVIEW AND IMPLICATIONS FOR VEGETATION AND CLIMATE RECONSTRUCTION DURING THE LATE PLEISTOCENE-HOLOCENE. In this review the results of Late Pleistocene-Holocene palynological studies from alluvial deposits in the Pampean region (PR) performed over the last ca. 30 years have been synthesized and discussed. Most vegetation reconstructions for that period have been derived from pollen analysis of alluvial deposits, although in some cases the results are controversial. The present review shows that palynological analysis from alluvial sequences is an important resource of qualitative and quantitative paleoenvironmental information for the PR, but also states that not all published pollen records are suitable for vegetation and climate reconstructions. The main causes of discrepancies between the results are the limited understanding of pollen fluvial transport, pollen preservation and taphonomy, and sedimentary facies control that could have altered the pollen records. The causes of the alteration of the pollen assemblages, although mainly related to repeated wetting-drying cycles, microbial degradation and possible control of facies, have scarcely been discussed in most works. This points the need for studies on differential pollen preservation and quantification of pollen transported by river water, paying more attention to the knowledge of taphonomic processes to improve the interpretation of fossil palynological records. Even so, the progress achieved recently have made possible to obtain significantly more precise and informative reconstructions of past vegetation and climate in the PR than was possible earlier.

Key words. Fluvial palynology. Taphonomy. Pollen preservation. Argentina.

EL ANÁLISIS palinológico de sucesiones aluviales ha sido utilizado para reconstruir la historia de la vegetación y del clima durante el Cuaternario Tardío en varias áreas del Hemisferio
Norte desde hace décadas. Los primeros trabajos se realizaron en la región semiárida del sudoeste de EEUU (e.g., Martin, 1963; Mehringer, 1967; Mehringer et al., 1967; Hall, 
1977) y en el este de Rusia (e.g., Grichuk, 1967 y referencias allí citadas).

En las últimas décadas el análisis palinológico de sucesiones aluviales se ha incrementado, especialmente en las Grandes Llanuras y el sudoeste de EEUU (Delcourt y Delcourt, 1980; Hall, 1981, 1985, 1989, 1995, 2010; Fall, 1987; Baker et al., 2000; Work et al., 2005), en el norte de China (e.g., Xu et al., 1996; Ma et al., 2004) y en algunos países de Europa (e.g., Bisernia y van Geel, 2005; Albert y Pokorný, 2012).
Los estudios pioneros en Argentina fueron realizados por D'Antoni et al. (1985) y Prieto (1989) en la región pampeana (RP). En los últimos 30 años numerosos investigadores han utilizado el análisis palinológico de sucesiones aluviales de esta región para reconstruir la vegetación e inferir las condiciones ambientales y climáticas, principalmente durante el Holoceno (Tab. 1; Fig. 1). En dos sucesiones aluviales (sitio 18 y 4, Fig. 1) se estudiaron únicamente los palinomorfos fúngicos (Borel et al., 2001) y otros componentes orgánicos ácido-resistentes (Grill et al., 2007),

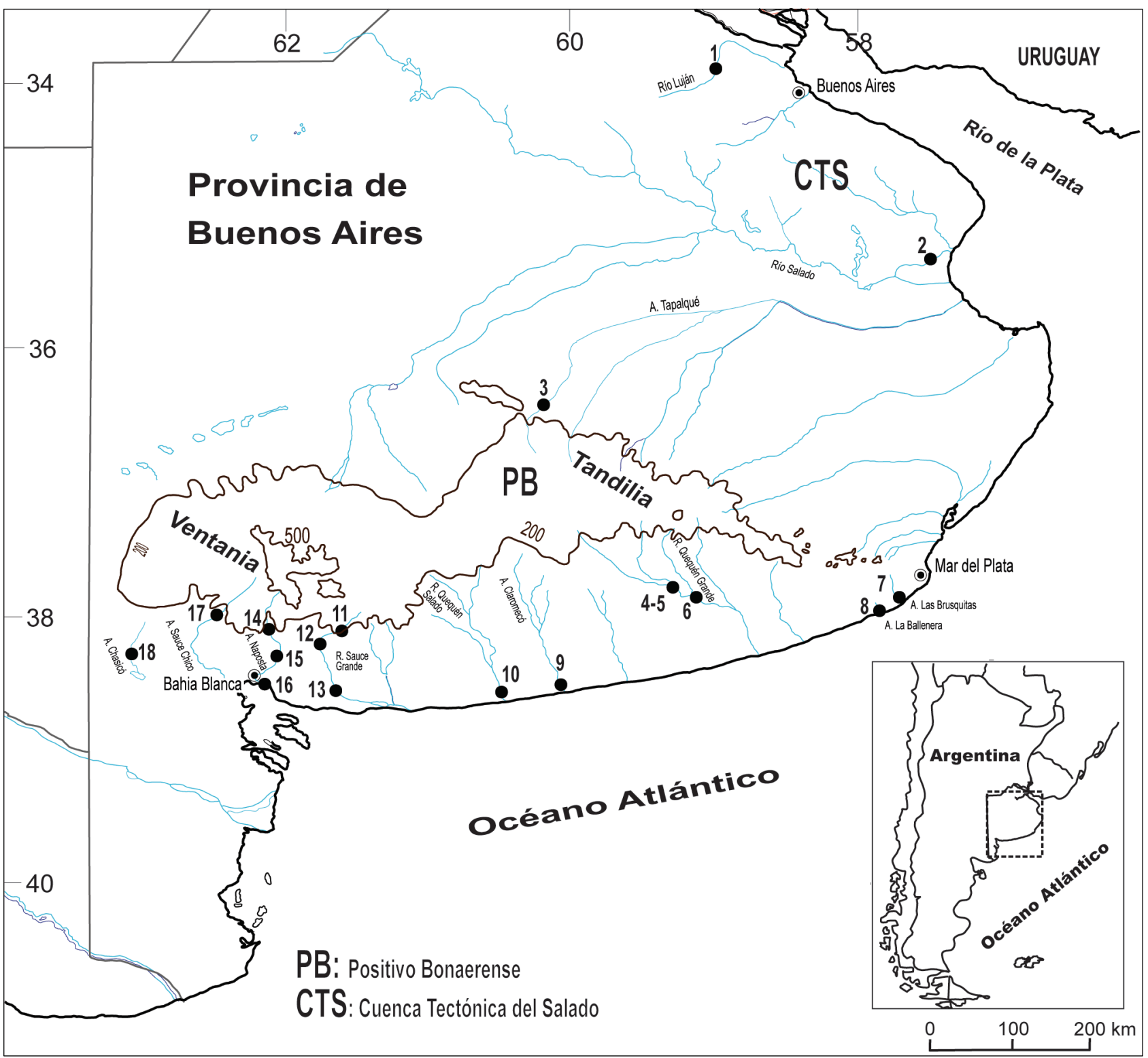

Figura 1. Mapa de ubicación de las sucesiones aluviales donde se realizaron los estudios palinológicos (ver referencias en Tabla 1). 
TABLA 1 - Sucesiones aluviales de la región pampeana donde se han realizado análisis palinológicos.

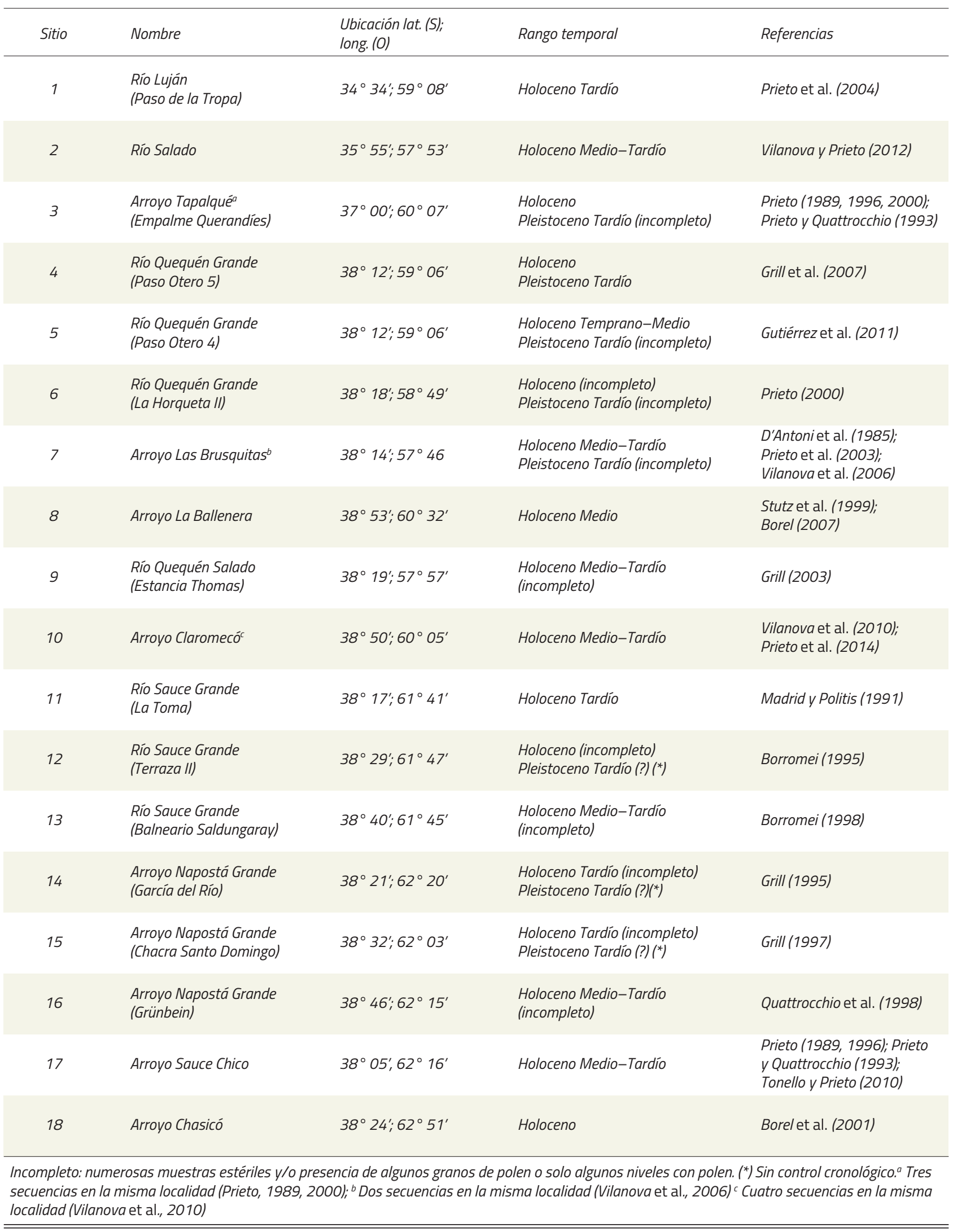


porque la mayoría de las muestras eran polínicamente estériles. Por otra parte, Prieto y Quattrocchio (1993) realizaron la única descripción y análisis de las briofitas y pteridofitas del Holoceno de la RP a partir de varias secuencias polínicas, algunas de las cuales provenían de depósitos aluviales (sitios 3 y 17, Fig. 1). En ese estudio se enfatizó que la información paleoambiental que brindaron las esporas de briofitas era de carácter local y se hipotetizó sobre la representación extra local y las posibles rutas migratorias de las pteridofitas durante el Holoceno.

Recientemente Rojo et al. (2012) iniciaron el análisis polínico de sucesiones aluviales del Holoceno Medio y Tardío en las regiones áridas-semiáridas del oeste de Argentina. A partir de esos análisis infirieron cambios locales de las comunidades vegetales de la llanura de inundación en respuesta a la dinámica fluvial del Arroyo La Estacada (33 S) durante ese tiempo.

La mayoría de los estudios palinológicos de depósitos aluviales de la RP provienen de afloramientos en los cursos colectores principales del Positivo Bonaerense y unos pocos de la Cuenca Tectónica del Salado (Fig. 1). Representan el material detrítico transportado y depositado por una corriente de agua principalmente en la llanura de inundación, en cuerpos de agua de tamaños variables en el valle y en la desembocadura, donde formaron ambientes estuáricos. Las sucesiones son estratigráficamente similares y muestran secciones semejantes como resultado de procesos sedimentarios discontinuos, indicados por superficies de erosión y desarrollo de suelos que señalan fases de relativa estabilidad (Fig. 2). Estas sucesiones representan el relleno sedimentario de los valles actuales, constituido principalmente por conglomerados y arenas basales a las que continúan depósitos arenosos y pelíticos y loess retrabajado por procesos fluviales del Pleistoceno Tardío (ca. 40.000$10.000{ }^{14} \mathrm{C}$ años AP). Para el Holoceno está representado por facies fluvio-lacustres, palustres y estuáricas que se acumularon en las áreas deprimidas de la paleotopografía preexistente, que presentan una geometría lenticular; y facies eólicas (Fig. 3). Durante el Holoceno Temprano y Medio dominó la sedimentación bioclástica (diatomitas o sedimentos diatomáceos y niveles con concentración de moluscos dulceacuícolas o estuarinos), durante el Holoceno Tardío dominó la sedimentación eólica y recientemente la aluvial (e.g., Fidalgo et al., 1973; Rabassa, 1989; Zavala y Quattrocchio, 2001; Prieto et al., 2004; Zárate, 2005; Fucks et al., 2010). La excavación de los cauces de los ríos y arroyos habría ocurrido con posterioridad a ca. 3000 años ${ }^{14} \mathrm{C}$ AP (Zárate et al., 2000; Vilanova et al., 2010) lo cual permitió la preservación y exposición fuera del área de incisión de un amplio conjunto de depósitos pre-incisión de varias edades que registran una historia detallada de los episodios de agradación durante el Pleistoceno Tardío-Holoceno.

Fall (1987) sugirió que el polen de depósitos aluviales no era confiable para la reconstrucción de paleoambientes. Sin embargo, la mayoría de los autores mencionados al inicio de esta revisión están en desacuerdo y reconocen que es una herramienta adecuada para realizar reconstrucciones de la vegetación local y regional y del clima del pasado; pero recomiendan que para que las interpretaciones sean más ajustadas se consideren (1) las facies sedimentarias y la configuración geomorfológica de los depósitos aluviales (Hall, 1989; Xu et al., 1996; Work et al., 2005) y (2) se entienda el papel de la tafonomía en la supuesta modificación de los conjuntos polínicos de estos depósitos (Brush y Brush, 1972; Fall, 1987; Albert y Pokorný, 2012).

Los estudios combinados de estratigrafía y análisis palinológico han contribuido a mejorar, precisar y profundizar la comprensión de los cambios observados en los ambientes aluviales, la vegetación y las condiciones ambientales y climáticas en la RP. Sin embargo, en ciertos casos los resultados son controvertidos (Prieto et al., 2009) y en consecuencia plantean varias cuestiones para discutir sobre el análisis palinológico de sucesiones aluviales en esta región. Por esta razón, en este trabajo se han sintetizado los resul-

Figura 2. Ejemplos de sucesiones aluviales en la región pampeana. 1, Arroyo Claromecó (sitio 10). 2, Río Quequén Salado (sitio 9). 3, Arroyo Las Brusquitas (sitio 7). 4, Río Quequén Salado. 5, Río Salado (sitio 2). 6, Arroyo Tapalqué (sitio 3). 7, Arroyo Sauce Chico (sitio 17). Fotografías: A.R. Prieto. Para detalles ver las referencias citadas en la Tabla 1. 

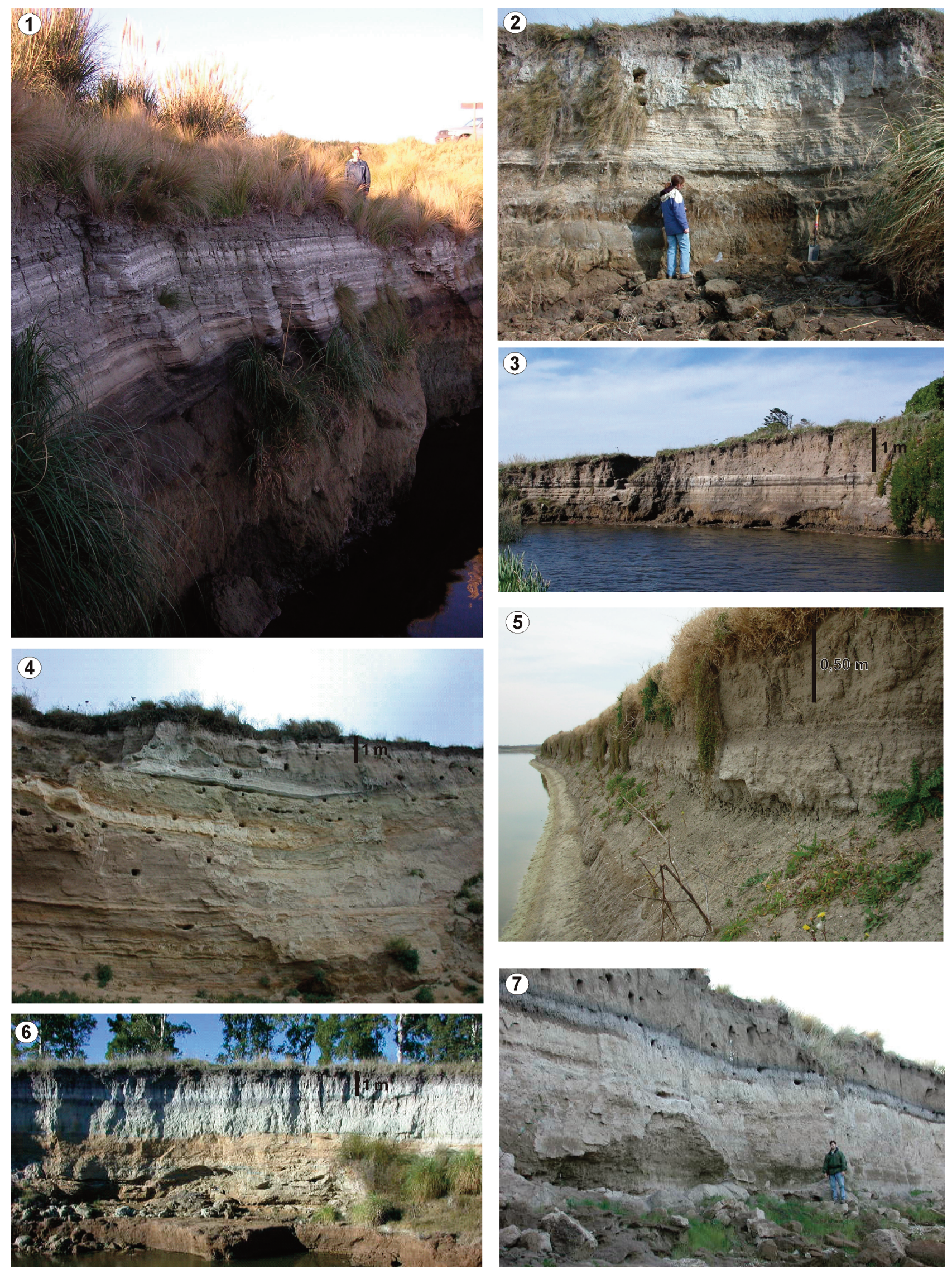
tados de ca. 30 años de estudios con los siguientes objetivos: (1) analizar el transporte fluvial, los procesos tafonómicos y el control de las facies sedimentarias en las posibles modificaciones de los registros polínicos de los depósitos aluviales; (2) evaluar la confiabilidad del contenido polínico de estos depósitos; y (3) discutir las potencialidades y limitaciones de los registros palinológicos de depósitos aluviales en los estudios de reconstrucción de la vegetación y del clima de la RP.
En este trabajo se utiliza el término "polínico" cuando el análisis incluyó el estudio del polen y de las esporas de pteridofitas y briofitas (Seppä, 2013) y el término "palinológico" cuando el estudio incluyó además a los palinomorfos no polínicos (van Geel, 2001), los dinoquistes y los acritarcos. La nomenclatura de las plantas vasculares sigue el catálogo del Instituto de Botánica Darwinion de Argentina (http://www.darwin.edu.ar) y el del Missouri Botanical Garden (http://www.tropicos.org/).

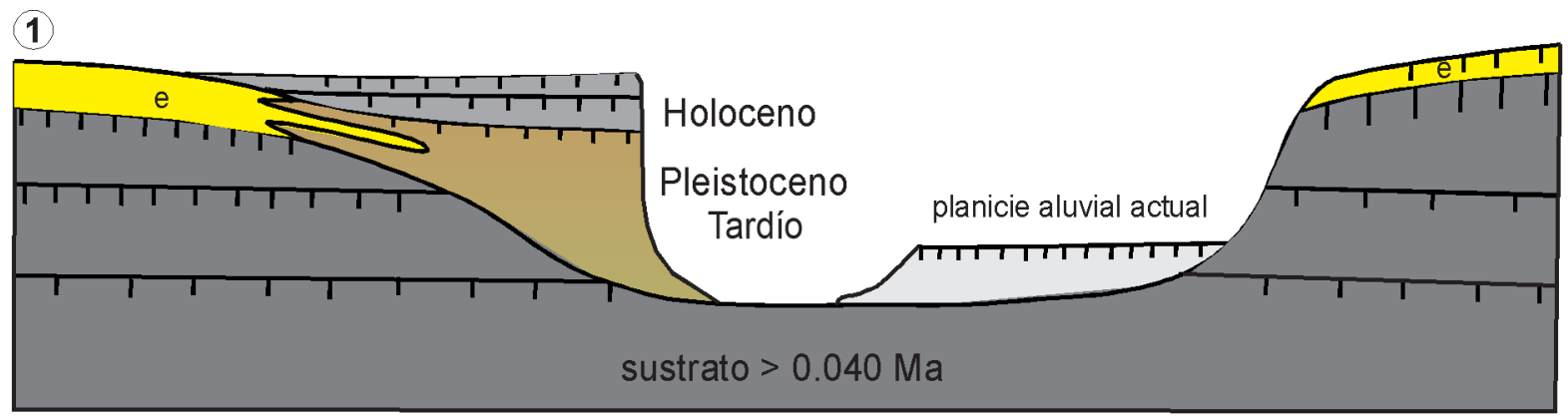

e: facies eólicas

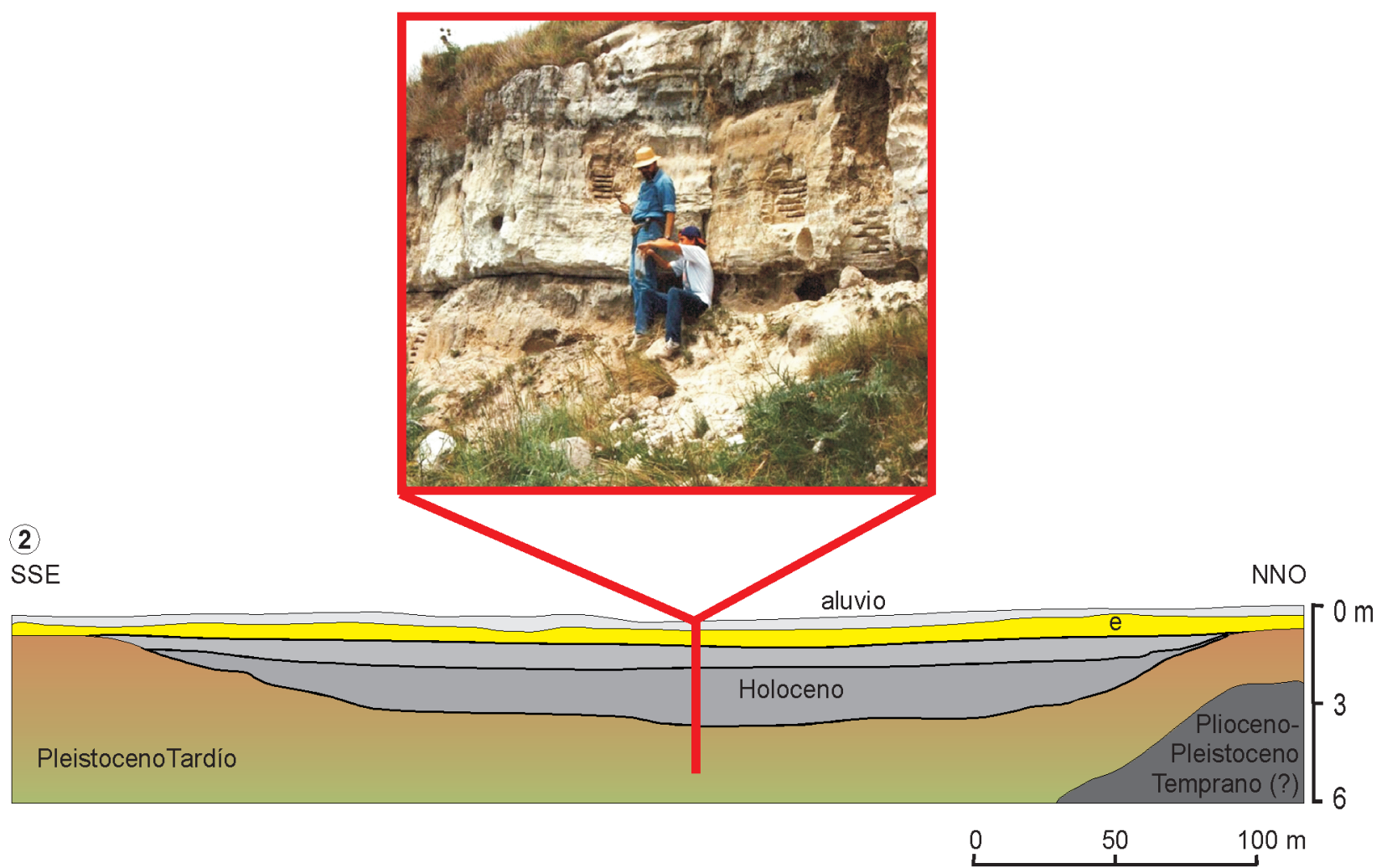

Figura 3. 1, Esquema simplificado de un valle fluvial en el Positivo Bonaerense (modificado de Zárate, 2005). 2, Geometría del sitio La Horqueta II (modificado de Zárate et al., 1998) indicado en rojo y en la fotografía la sección estratigráfica estudiada. 


\section{TRANSPORTE FLUVIAL DEL POLEN}

El polen transportado fluvialmente es una importante fuente de palinomorfos contenidos en los depósitos aluviales y los palinólogos han tenido diferentes opiniones sobre el polen aluvial. Por tal motivo, han examinado el polen suspendido en el agua en numerosos ríos y arroyos (e.g., Catto, 1985; Chmura y Liu, 1990; Traverse, 1992; Smirnov et al., 1996; Xu et al., 1996; Chmura et al., 1999; Brown et al., $2007,2008)$ y el incluido en muestras de sedimento superficial (e.g., Grichuk, 1967; Solomon et al., 1982; Fall, 1987; Xu et al., 1996; Moss et al., 2005; Rojo et al., 2012), para evaluar las fuentes fluviales del polen. Estos últimos estudios se han realizado en diferentes ubicaciones dentro del ambiente fluvial como la llanura de inundación, las barras, el lecho, los albardones e incluso en la llanura aluvial fuera del río, tanto en grandes ríos que atraviesan diferentes zonas geográficas como en tributarios localizados dentro de una única zona.

Algunos estudios han mostrado que la composición taxonómica de los conjuntos polínicos transportados por el agua de los ríos no tendría el mismo efecto sobre aquellos involucrados en el transporte por el viento, particularmente sobre el efecto que el transporte por el agua tendría en la clasificación del polen. Brown (1985), Catto (1985), Fall (1987) y Xu et al. (1996) han argumentado que el transporte fluvial tiende a clasificar hidráulicamente a los granos de polen por tamaño, concentrando preferentemente a unos como componentes de la fracción arena y a otros con la fracción limo-arcilla. Estos hallazgos son consistentes en general con los modelos experimentales de transporte de polen en un canal artificial y, si bien el tamaño es el factor más importante en la clasificación, también están involucradas la forma y/o la densidad de los granos de polen (Brush y Brush, 1972; Holmes, 1990). Contrariamente, SoIomon et al. (1982), Hall (1989), Chmura y Liu (1990), Campbell y Chmura (1994), Smirnov et al. (1996) y Moss et al. (2005), han concluido que la clasificación de los granos de polen por el transporte fluvial no es un factor significativo, que los conjuntos polínicos son independientes de la textura sedimentaria y que no es esperable que el transporte fluvial provoque distorsiones de los conjuntos polínicos en los sedimentos. Por lo tanto, los granos de polen transportados fluvialmente reflejarían fielmente a las comunidades vegetales y podrían utilizarse en la reconstrucción de la vegetación y del clima del pasado.

Estas conclusiones no son extrapolables de manera directa a todas las regiones y depósitos aluviales e incluso las interpretaciones de los registros polínicos fósiles a partir de estos estudios actualísticos difieren para un mismo ambiente (e.g., región semiárida del sudoeste de EEUU: Fall, 1987; Hall, 1989). Este tipo de estudios no se ha realizado en la RP y si bien algunos de los resultados pueden utilizarse para la interpretación de los conjuntos polínicos fósiles provenientes de los depósitos aluviales, también es esperable diferencias debido a que las regiones donde se obtuvieron esas conclusiones tienen comunidades vegetales y condiciones climáticas diferentes a la RP. Sin embargo, este conocimiento ha sido usualmente descuidado en los estudios de las secuencias polínicas fósiles de esta región. Solamente en el registro palinológico Arroyo Sauce Chico, ubicado en el curso medio del arroyo homónimo (sitio 17, Fig. 1), se explicó la presencia de las pteridofitas Cheilanthes/ Pellaea spp., Notholaena squamosa (Gillies y Hook. ex Grev.) Hook. y Baker, Anemia tomentosa (Sav.) Swartz, Doryopteris sp. y Polypodium sp. como resultado del transporte fluvial de estas esporas desde la región serrana de Ventania (Fig. 1), donde nace el arroyo y se desarrollan esas plantas (Prieto y Quattrocchio, 1993). Esto sugiere la posibilidad de que el agua transporte también polen desde las cabeceras, el cual contribuiría al conjunto polínico de los sedimentos aluviales aguas abajo.

Por otra parte, el transporte y la suspensión diferencial explicarían la heterogeneidad de los espectros polínicos provenientes de los mismos depósitos aluviales del Holoceno del Arroyo Napostá Grande (Grill, 1995, 1997; sitios 14 y 15, Tab. 1), en lugar de atribuirle una causa climática. La heterogeneidad está sugerida por señales polínicas diferentes y tendencias particulares en cada registro y ha dificultado la comparación entre las secuencias polínicas y la interpretación de los cambios de la vegetación a escala local como regional. Además, debería tenerse en cuenta un posible control de facies para explicar la heterogeneidad de cada registro, los cuales pueden diferir significativamente con diferentes facies (Xu et al., 1996). Este control no ha sido contemplado, aun cuando la variabilidad facial es considerable en esas sucesiones (Quattrocchio et al., 2008). 
En los estudios actualísticos el polen de Poaceae fue asociado con el tamaño limo y correlacionado con ambientes de baja energía, y el de Cyperaceae y Chenopodiaceae con el tamaño arena y vinculados con ambientes de alta energía (e.g., Brown, 1985; Catto, 1985; Fall, 1987). Por el contrario, en algunas secuencias de la RP el polen de Cyperaceae se presenta con las mayores frecuencias y concentraciones en los limos orgánicos (e.g., Stutz et al., 1999; Vilanova et al., 2010) en una relación inversa a la obtenida en los estudios actualísticos. En general, la selección hidráulica por tamaños no es evidente en las secuencias polínicas de la RP, posiblemente porque el rango de tamaños de los granos de polen es equivalente al limo mediano hasta arena muy fina, litologías predominantes en los depósitos aluviales estudiados.

El resultado del transporte fluvial del polen debería ser considerado cuidadosamente en la reconstrucción de la vegetación. La influencia que el mismo puede tener sobre la representación polínica aún no está completamente entendida, en particular en lo referente a la forma en que este transporte afecta a la depositación y preservación del polen y, por lo tanto, a la representación polínica en un registro fósil (Moss et al., 2005).

\section{PRESERVACIÓN POLÍNICA Y TAFONOMÍA}

Aunque la preservación del polen es un factor importante que afecta la correcta interpretación de un espectro polínico (e.g., Bryant y Holloway, 1983; Campbell, 1991; Bunting y Tipping, 2000; Tipping, 2000), ha sido frecuentemente ignorada en las investigaciones de depósitos aluviales de la RP. Sin embargo, casi todos los conjuntos polínicos de las sucesiones aluviales involucran aspectos de la preservación del polen en cierta medida. La preservación polínica depende de muchos factores complejos que podrían agruparse en tres categorías (Bryant y Holloway, 1983): (1) las características intrínsecas del polen, tales como la composición química de la pared, el tamaño del grano y la estructura y escultura de la exina (Havinga, 1964, 1967, 1984); (2) los factores ambientales como la oxidación (pH y Eh) (Dimbleby, 1957; Tschudy, 1969; Bryant et al., 1994; Twiddle y Bunting, 2010), la temperatura y humedad fluctuantes (Bryant y Holloway, 1983; Campbell y Campbell, 1994), la acción de hongos y bacterias (Havinga, 1970; Elsik,
1971) y la (bio) química (Havinga, 1964) y (3) la degradación mecánica (abrasión) (Catto, 1985; Fall, 1987), aunque en un experimento de laboratorio se encontró que la evidencia de daños causados por factores mecánicos era escasa (Campbell, 1991).

En varios trabajos de la RP se ha reconocido que la mala preservación y la destrucción diferencial del polen es recurrente en las sucesiones aluviales (e.g., Prieto, 2000; Borel et al., 2001; Prieto et al., 2004; Grill et al., 2007; Quattrocchio et al., 2008; Vilanova et al., 2010), sin embargo, los posibles factores tafonómicos que las han causado han sido escasamente discutidos. Prieto (2000) explicó la preservación diferencial en algunas muestras del sitio 3 (Fig. 1), que presentaban hasta el 70\% de los granos de polen deteriorados, como producto de la actividad microbiana en función del patrón de alteración que presentaba la pared de los granos (Havinga, 1970, 1984). Esas muestras no fueron consideradas en los análisis porque el conjunto polínico no era confiable. La actividad microbiana también fue propuesta por Prieto et al. (2004) como el factor más probable responsable de la degradación polínica en una secuencia del Río Luján (sitio 1, Fig. 1) en lugar de un pH alto, basado en que la falta de correlación entre los porcentajes de $\mathrm{CO}_{3} \mathrm{Ca}$, los porcentajes de polen degradado y los valores de concentración polínica total, no sustentaban una relación con un ambiente alcalino. Una conclusión similar fue observada por Bryant et al. (1994) para muestras fósiles del sudoeste norteamericano.

Vilanova et al. (2006) han relacionado la mala preservación de los palinomorfos en algunos niveles ente ca. 3900 y $3000{ }^{14} \mathrm{C}$ años AP en el Arroyo Las Brusquitas (sitio 7, Fig. 1) con la desecación periódica y el retrabajo asociados a una zona supralitoral.

Grill et al. (2007) realizaron una clasificación de las clases de deterioro de la materia orgánica palinológica y de los posibles procesos responsables que eliminaron casi por completo el polen de las muestras de sedimento de una sucesión aluvial (sitio 4, Fig. 1). Estos autores atribuyeron como la causa principal de la destrucción del polen a un $\mathrm{pH}$ alto, pero también señalaron a otros factores como la oxidación química y bioquímica y el daño mecánico, sin explicar en qué casos y cómo afectaron al polen. Debido al escaso contenido de polen preservado, el análisis palinofacial que 
realizaron está sesgado y la interpretación no reflejaría de manera representativa a la vegetación local ni regional durante el lapso considerado, dando lugar a interpretaciones paleoecológicas erróneas.

La ausencia casi total de polen en muchos de los depósitos aluviales del Pleistoceno Tardío y el Holoceno Temprano podría explicarse por el descenso del nivel freático y repetidos ciclos de hidratación-desecación como consecuencia de la excavación de los cauces y las fluctuaciones del nivel freático, respectivamente (e.g., Vilanova et al., 2010). Hasta el momento de la disección, ocurrida con posterioridad a ca. $3000{ }^{14} \mathrm{C}$ años AP, el nivel freático era alto y en consecuencia es factible conjeturar que durante ese lapso el sustrato estuvo en condiciones de saturación y por lo tanto reductoras (bajo Eh). Con posterioridad a la incisión, el depósito sedimentario que contiene al polen sin-depositacional pasó a ser un sustrato aireado en un ambiente oxidante y con cambios en el Eh (Tonello et al., 2002). Un Eh alto ocasiona un deterioro químico de la exina y una destrucción diferencial o total de los granos de polen (Tschudy, 1969), así como los ciclos repetidos de hidratación-desecación son una de las causas principales de la rápida degradación del polen (Holloway, 1989; Campbell y Campbell, 1994). Por lo tanto, la ausencia o mala preservación sería el resultado de alteraciones post-depositacionales, como se ha registrado en otros depósitos aluviales (e.g., Hall, 1977, 1995) aunque no se puede descartar un deterioro sin-depositacional. Estas explicaciones resultan más parsimoniosas que aquellas que atribuyeron la ausencia de polen en los registros del Pleistoceno Tardío a condiciones extremadamente áridas (Grill, 1995; Quattrocchio et al., 2008), a una cobertura vegetal reducida por condiciones ambientales severas (Grill, 1995) y/o a la ausencia de cobertura vegetal (Grill et al., 2007), en contradicción con los numerosos resultados palinológicos obtenidos en depósitos de zonas áridas (e.g., Horowitz, 1992 y referencias allí citadas).

Aunque los niveles freáticos pueden haber estado muy bajos en los valles aluviales durante el Holoceno Tardío, los depósitos también pueden haber tenido una larga historia de percolación de agua. A estos procesos post-depositacionales que pueden alterar los conjuntos polínicos, que generalmente involucran la destrucción y la pérdida por oxidación del polen, se le deben añadir los procesos de meteorización y formación de suelos, ocurridos en varios momentos del Holoceno (e.g., Zárate, 2005). La génesis de los suelos ocurrió durante y con posterioridad a los procesos de sedimentación (e.g., Zárate et al., 2000). La superficie enterrada de esos paleosuelos suele estar indicada en los registros polínicos por un incremento de la concentración polínica total, una disminución de los granos deteriorados y un aumento de Glomus, un hongo micorriza de los suelos (e.g., Dimbleby, 1985; Vilanova et al., 2010).

Los procesos tafonómicos son evidentes en algunas secuencias polínicas del Holoceno, las que presentan numerosos niveles estériles y/o con escasos granos de polen (indicados en los diagramas polínicos con el término "presencia" e.g., Grill, 1995, 2003; Borromei, 1998). Estos resultados sugieren que ha existido una eliminación diferencial de ciertos tipos polínicos y en consecuencia se ha incrementado la importancia aparente de los tipos más resistentes. De esta forma, con el aumento del deterioro se introduce un sesgo en favor de aquellos tipos polínicos que son más fáciles de reconocer por el analista, como por ejemplo Chenopodiaceae, Brassicaceae y Asteraceae, como se observa en varias de las secuencias analizadas.

\section{Modificaciones y alteraciones de los espectros palinoló- gicos de depósitos aluviales de la región pampeana: una explicación posible a partir de un caso de estudio}

Para ejemplificar las posibles modificaciones y alteraciones que podrían haber afectado a los espectros palinológicos de los depósitos aluviales de la RP, se presenta como caso de estudio los análisis palinológicos realizados en tres afloramientos del Río Quequén Grande (Fig. 1) en un tramo de $20 \mathrm{~km}$. Se discuten las posibles causas de la mala preservación y/o ausencia de polen en relación con procesos post y sin-depositacionales, el transporte fluvial y el control de facies.

El primero de los análisis se realizó en La Horqueta II (sitio 6, Tab. 1) a partir de una sección en la margen derecha del mencionado río, representativa del registro aluvial del centro-sur de la RP (Zárate et al., 2000) que abarcó desde el Pleistoceno Tardío (ca. 19.000-10.000 ${ }^{14} \mathrm{C}$ años AP) hasta la actualidad (Prieto, 2000). La sección datada entre 10.000 y ca. $3000{ }^{14} \mathrm{C}$ años AP es una diatomita que presenta una geometría lenticular y subyace a un depósito de origen 
eólico modificado por pedogénesis y enterrado por aluvio actual (Fig. 2). Los únicos conjuntos polínicos se recuperaron de siete niveles de la base de la diatomita (10.270-9000 ${ }^{14} \mathrm{C}$ años AP) y en dos del Pleistoceno (Prieto, 2000). Los espectros están dominados por polen de Brassicaceae, generalmente formando agregados (masas cohesivas de dos o más granos de polen del mismo taxón). Se interpretó que la sobrerrepresentación de este taxón fue originada por una rápida depositación local en relación con un ambiente disturbado, en lugar de un registro de preservación polínica diferencial (Prieto, 2000). Los demás niveles fueron estériles o solo se recuperaron escasos granos de polen, algunos mal preservados, los que no fueron utilizados para realizar reconstrucciones de la vegetación, ya que las sumas polínicas no eran estadísticamente representativas y la confiabilidad del registro estaría fuertemente cuestionada. Bajas concentraciones y mala preservación polínica también se han obtenido en niveles de diatomitas en diferentes sitios de Nuevo México y Texas, las que fueron atribuidas a procesos post-depositacionales (Hall, 1995).

La segunda sección está localizada en Paso Otero 5 (sitio 4, Tab. 1), $20 \mathrm{~km}$ aguas arriba y abarcó desde el final del Pleistoceno Tardío (> $10.440{ }^{14} \mathrm{C}$ años AP) hasta la actualidad (Grill et al., 2007). Se trata de la misma sucesión aluvial identificada aguas abajo por Zárate et al. $(1998,2000)$ con algunas diferencias faciales (Johnson et al., 2012). Prácticamente todas las muestras fueron polínicamente estériles o contenían escasos granos de polen para realizar recuentos y estaban dominadas por esporas de hongos (Grill et al., 2007), indicando que habría ocurrido desde una preservación diferencial hasta la destrucción total del polen.

La tercera sección está ubicada $2 \mathrm{~km}$ aguas abajo de la anterior en Paso Otero 4 (sitio 5, Tab. 1). Ambos sitios forman parte de la localidad arqueológica homónima y los depósitos aluviales son semejantes. La secuencia polínica abarcó el lapso $10.500-2280{ }^{14} \mathrm{C}$ años AP y solo presenta escasas muestras estériles en la base del Holoceno (Gutiérrez et al., 2011). Los espectros polínicos están dominados por Asteraceae, Brassicaceae y Poaceae acompañados por Chenopodiaceae y Apiaceae, todos en proporciones altamente variables. Brassicaceae exhibe proporciones mayores al $50 \%$ en varios niveles, entre ca. 6500 y $4500{ }^{14} \mathrm{C}$ años AP y Apiaceae alcanza hasta 58 y $65 \%$ en dos niveles data- dos en $8913 \pm 49$ y $4561 \pm 41^{14} \mathrm{C}$ años AP, respectivamente, y se presentan en agregados. La sobrerrepresentación de ambos fue relacionada con actividades humanas, sin embargo resultaría de una depositación local. Posiblemente el polen provendría de plantas acuáticas flotantes, donde Brassicaceae representaría a plantas semejantes al berro (Nasturtium sp.) (Vilanova et al., 2010) y Apiaceae a Hydrocotyle sp., desarrolladas en ambientes de aguas someras, vegetados y con baja o nula velocidad de corriente como lo sugieren el polen de otras hidrofitas, las diatomeas, los fitolitos y los palinomorfos no polínicos (Gutiérrez et al., 2011).

La disparidad en los resultados polínicos entre los tres registros no fue discutida en este último trabajo, aun cuando todos los registros provienen de los mismos depósitos aluviales y están ubicados muy próximos unos con otros. La preservación polínica diferencial y un control de facies, principalmente para las sucesiones del Holoceno son evidentes.

Según Johnson et al. (2012) existen algunas diferencias depositacionales entre las áreas de Paso Otero y La Horqueta que indican facies diferentes, con una sedimentación episódica y precipitación de carbonatos en la primera y aguas más o menos estancadas y depositación de diatomeas en la segunda. Estas diferencias también se reflejan en los estudios diatomológicos que sugieren que en La Horqueta II (Zárate et al., 1998) los cuerpos de agua dulce-salobre fueron más profundos y menos vegetados que en Paso Otero 5 (Gutiérrez et al., 2011). Probablemente, un cambio en el pH como respuesta a un aumento en el contenido de carbonatos, habría cambiado la química del agua favoreciendo la preservación de huesos en el primer sitio y la formación de diatomita en el segundo (Johnson et al., 2012). Sin embargo, esto no explicaría totalmente las diferencias en la preservación polínica entre unas y otras secuencias.

La presencia de carbonato en las sucesiones aluviales durante el Holoceno Medio podría reflejar altas tasas de evaporación con la consecuente pérdida de $\mathrm{CO}_{2}$ debido a un incremento de la temperatura del agua superficial, o una reducción de las precipitaciones que originaría un enriquecimiento post-depositacional del carbonato (Prieto et al., 2004). Ambos casos tendrían su contraparte en cambios del nivel freático que ocasionarían ciclos de hidratación-dese- 
Perfil 1

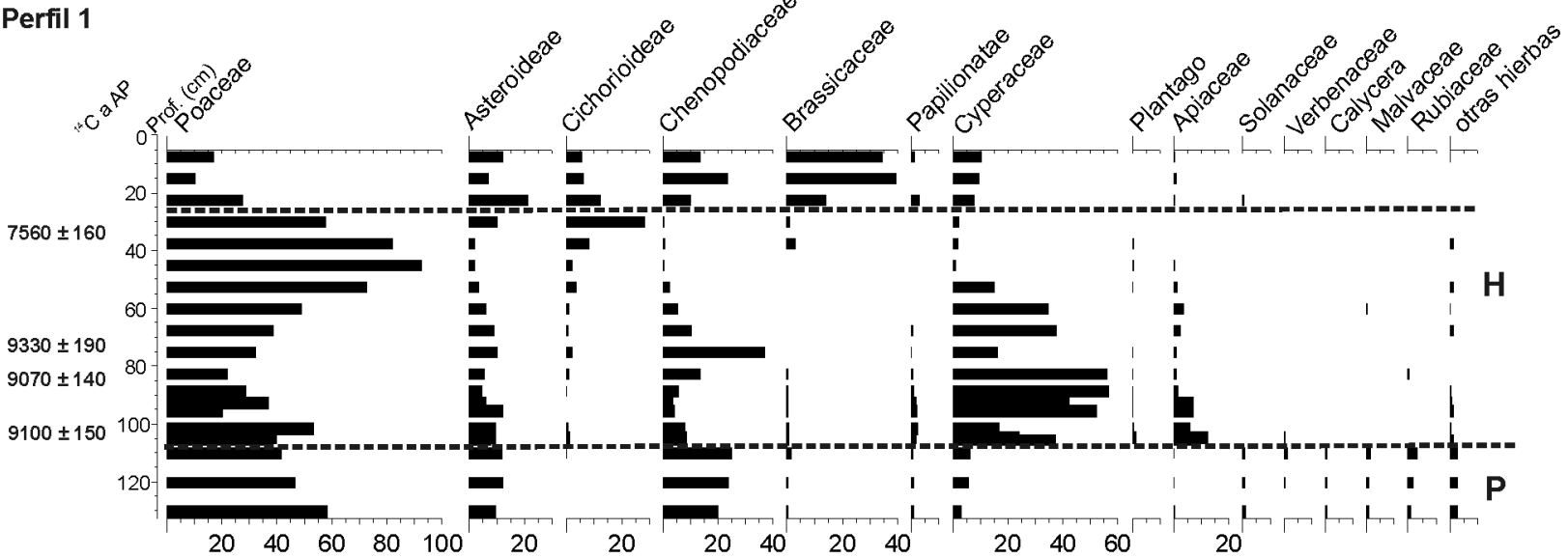

Perfil 2

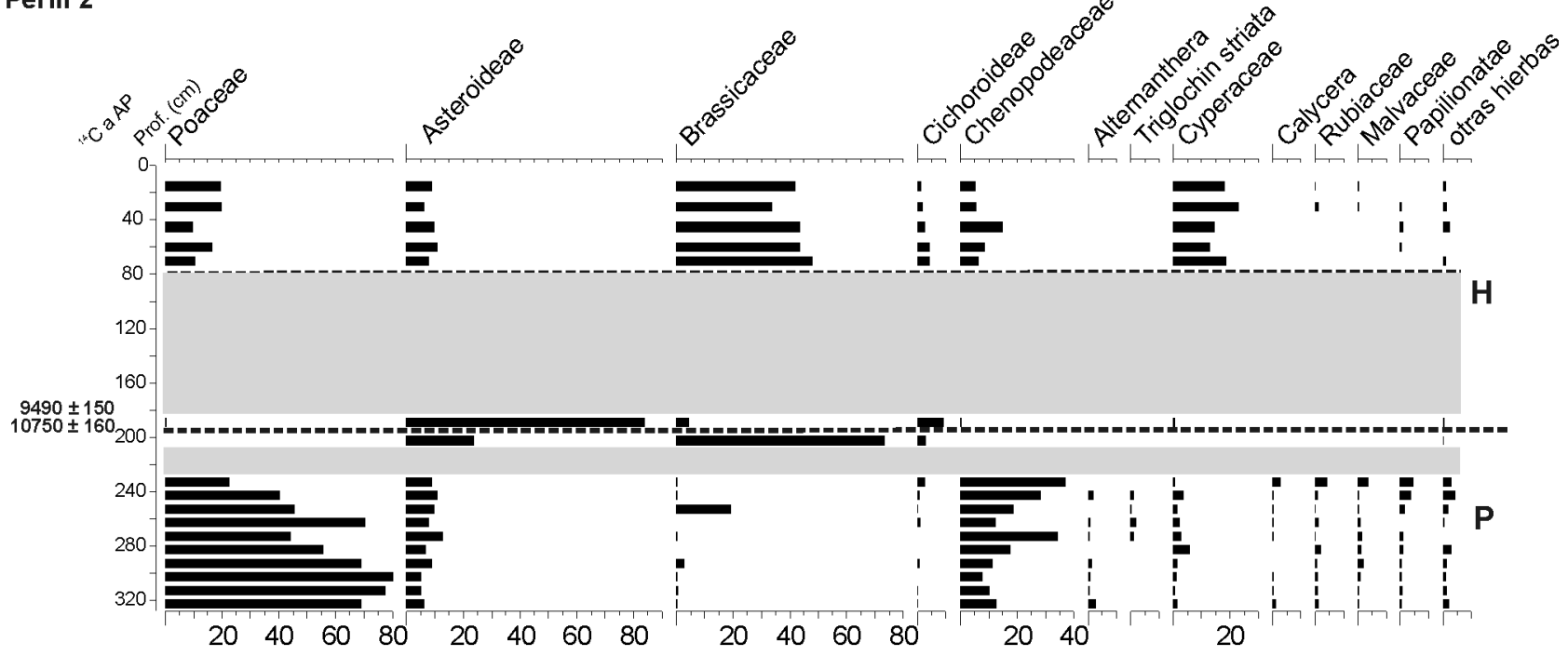

Perfil 3

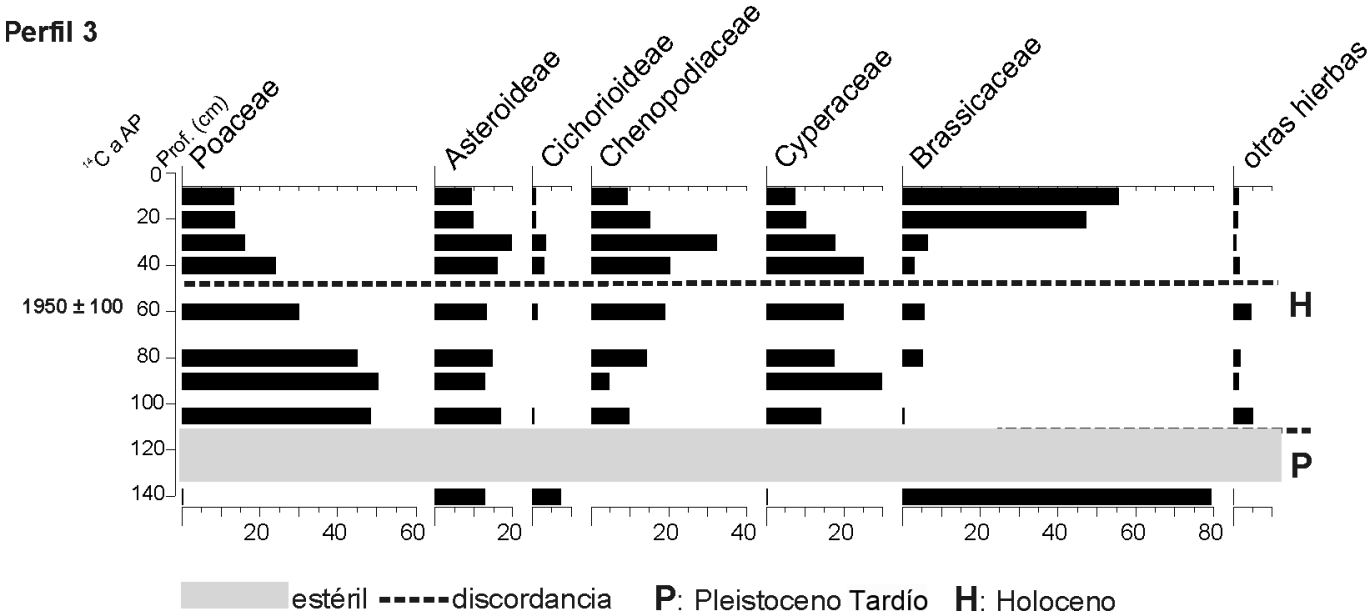

Figura 4. Diagramas polínicos en porcentajes de tres secciones estratigráficas en Empalme Querandíes, Arroyo Tapalqué (sitio 3, Fig. 1). Las secuencias se muestrearon en una sección transversal de 14 m (modificado de Prieto, 1989; 2000). 
NO

Perfil 1

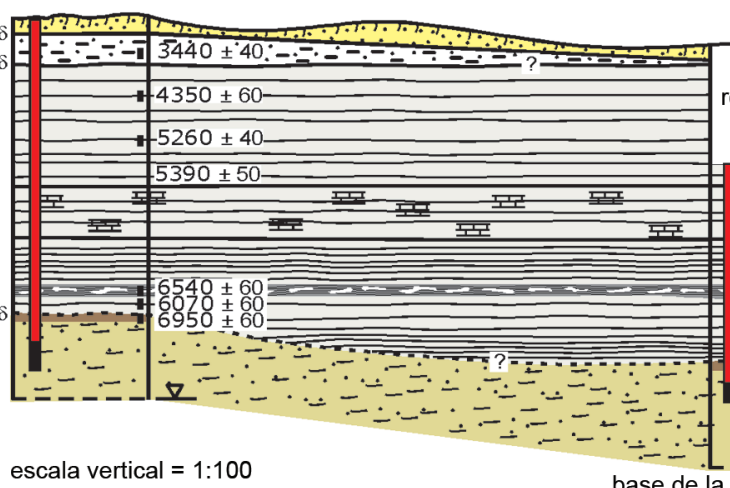

escala vertical $\mathbf{=} 1: 100$

secuencias palinológicas analizadas:
Arroyo Claromecó

Perfil 2

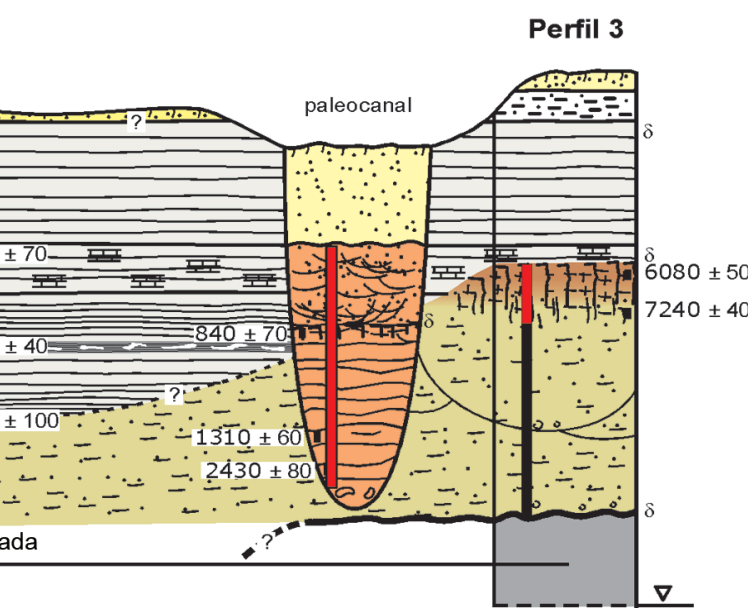

Figura 5. Sección general transversal de la estratigrafía del Pleistoceno Tardío-Holoceno del Arroyo Claromecó (sitio 10, Fig. 1) (modificado de Vilanova et al., 2010).

cación que podrían degradar la pared de los granos y causar la destrucción del polen (Bryant y Holloway, 1983; Campbell y Campbell, 1994). Si los depósitos pueden retener suficiente humedad durante esos ciclos, podrían prevenir la desecación completa y los efectos de la hidratación-desecación sobre el polen podrían no ser tan severos (Bryant et al., 1994) y esto explicaría la existencia de niveles con y sin polen.

Por otra parte, es notable que la secuencia que tiene el registro polínico con buena preservación (Paso Otero 4) sea la misma donde se han preservado huesos, sugiriendo que un $\mathrm{pH}$ alto no es necesariamente la principal causa de la destrucción del polen, como fue propuesto por Grill et al. (2007). Además, la diatomita puede haber tenido una larga historia de percolación de agua, a diferencia de los otros depósitos más arcillosos. En consecuencia, los ciclos de hidratación-desecación serían la principal causa para explicar la preservación diferencial del polen.

Es necesario resaltar que las secuencias palinológicas Paso Otero 4 y 5 (Tab. 1), separadas por 2 km y provenientes de la misma sucesión aluvial, muestran diferencias notables, que deberían tenerse en cuenta en la selección de secciones estratigráficas de depósitos aluviales para realizar estudios palinológico en futuros trabajos. El conocimiento de la geometría del cuerpo sedimentario y de la variabilidad facial de los depósitos aluviales permitirá se- leccionar varios perfiles estratigráficos a lo largo de una sucesión aluvial. Los análisis palinológicos de estas sucesiones brindarán una información espacio-temporal más ajustada y confiable para realizar reconstrucciones de la vegetación y el ambiente del pasado, como lo ejemplifican los trabajos de Prieto (1989, 2000) y Vilanova et al. (2010) (Figs. 4, 5 y 6).

\section{POTENCIALIDADES Y LIMITACIONES DE LOS REGIS- TROS PALINOLÓGICOS DE DEPÓSITOS ALUVIALES EN LOS ESTUDIOS DE RECONSTRUCCIÓN DE LA VEGETA- CIÓN Y EL CLIMA EN LA REGIÓN PAMPEANA}

\section{Reconstrucción de la vegetación}

La mayor parte de las reconstrucciones de la vegetación de la RP para el Pleistoceno Tardío-Holoceno han derivado del análisis polínico de sucesiones aluviales. La introducción de técnicas numéricas multivariadas aplicadas a los datos de polen y vegetación actual de esta región han ayudado a los palinólogos a entender la relación entre el polen y las comunidades de plantas (e.g., Stutz y Prieto, 2003; Tonello y Prieto, 2003, 2008; Vilanova y Prieto, 2012) y a ajustar las interpretaciones de los conjuntos polínicos fósiles de las sucesiones aluviales (Prieto, 1996, 2000; Vilanova et al., 2006; Vilanova y Prieto, 2012). Sin embargo, en la mayoría de los trabajos las interpretaciones se han basado principalmente 

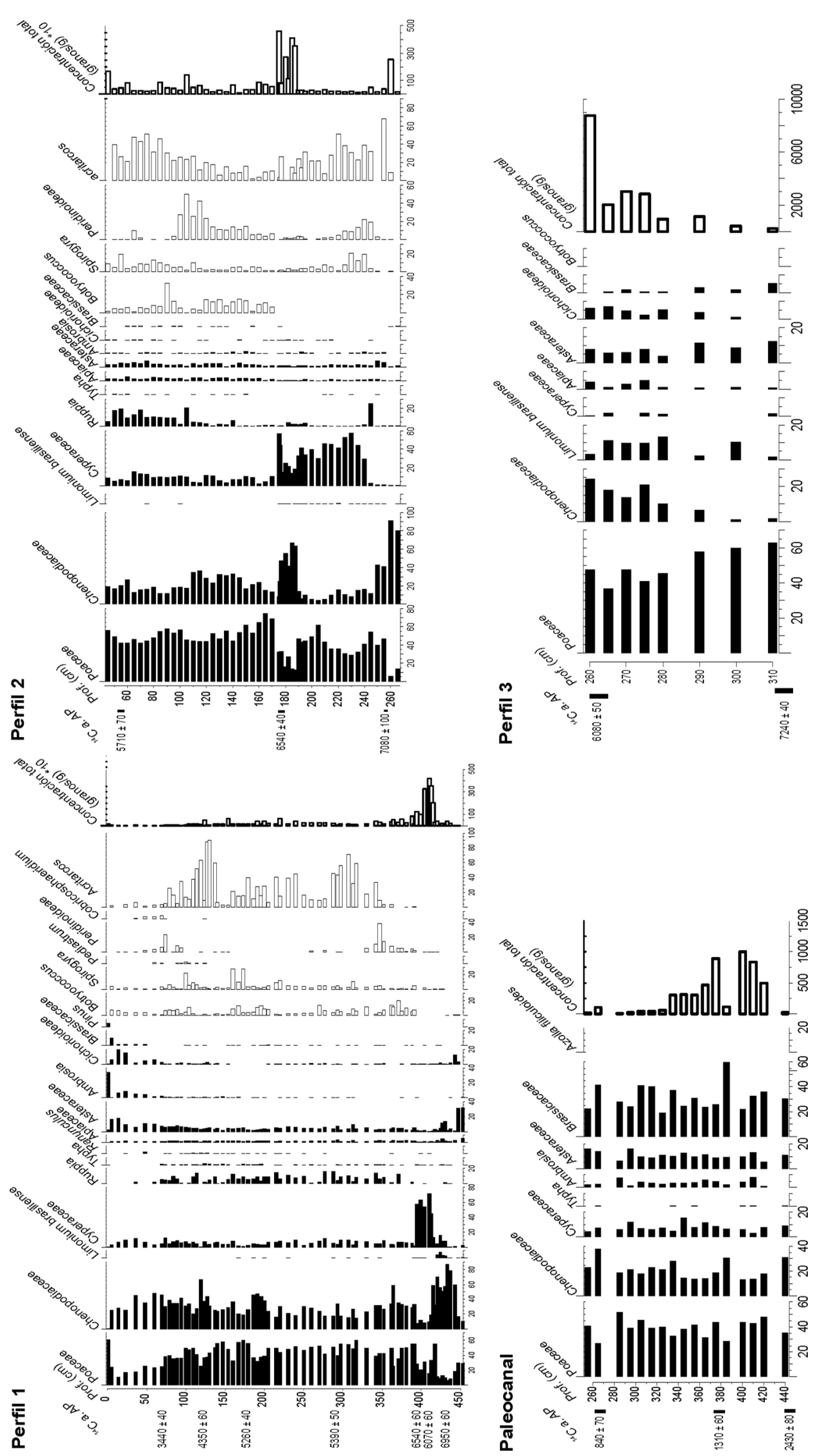
en el supuesto de que los porcentajes polínicos reflejan la vegetación local y regional, utilizando el polen fósil como un registro proxy directo de la vegetación del pasado sin considerar los sesgos ocasionados principalmente por los procesos de formación de los depósitos aluviales.

A diferencia de lo que ocurre para el Holoceno, la reconstrucción de la vegetación para el Pleistoceno Tardío de la RP proviene exclusivamente del análisis polínico de sucesiones aluviales. Sin embargo, la mayoría de las secuencias tienen escasos niveles con polen y baja diversidad, probablemente debido a los procesos tafonómicos explicados anteriormente. Registros polínicos completos son raros y están representados solamente por una de las secuencias del Arroyo Tapalqué (Perfil 2, Fig. 4) y otra del Río Sauce Grande (sitio 12, Tab. 1). Estos sugieren que entre ca. 16.000 y 12.000 años cal AP en amplias áreas del centro y sudoeste de la actual RP se desarrollaron pastizales psammófilos asociados con elementos arbustivos en el sudoeste (Prieto, 2000). En las otras secuencias, los escasos niveles con polen, algunos datados en $36.130 \pm 620{ }^{14} \mathrm{C}$ años AP (Prieto et al., 2003) y 19. $840 \pm 310{ }^{14} \mathrm{C}$ años AP (Prieto, 2000) y otros sin datar (e.g., Borromei, 1995; Grill, 1995; Gutiérrez et al., 2011), están representados principalmente por Brassicaceae, que alcanza proporciones de hasta $80 \%$ del registro, acompañada por Poaceae y Asteraceae y/o Chenopodiaceae, en proporciones variables. Cuando el polen de Brassicaceae y Asteraceae aparece en altas proporciones y formando agregados, ha sido interpretado como originado por una rápida depositación local en relación con un ambiente disturbado (Prieto, 1989, 1996; Borromei, 1995; Grill, 1997). El disturbio se relacionó con episodios de inundaciones rápidas y repentinas en depresiones de la llanura de inundación, causados por eventos inusuales de precipitación (Prieto, 2000). La analogía se realizó por comparación con los registros de Brassicaceae en tiempos históricos (Fig. 4), donde este taxón, en altos porcentajes, aparece en asociación con polen de otras especies ruderales y de árboles exóticos, como Eucalyptus spp. y Pinus spp., correspondientes a plantaciones que se iniciaron a finales del siglo XIX en la RP, indicando un disturbio, en este caso de origen antrópico (e.g., Prieto et al., 2004; Quattrocchio et al., 2008).

En síntesis, los registros polínicos de las sucesiones aluviales del Pleistoceno Tardío muestran numerosos nive- les estériles y están escasamente datados, como para realizar una reconstrucción comprensiva de la historia de la vegetación de la RP y hacer buenas correlaciones con otras secuencias de Argentina para ese tiempo.

Los depósitos aluviales del Holoceno muestran en general excelentes registros palinológicos, con buena resolución temporal (Tab. 1; Fig. 6), que han permitido realizar reconstrucciones de la vegetación local y regional y del ambiente.

Con las esperables variaciones locales, las secuencias del Holoceno Temprano muestran que las señales polínicas están marcadas por el incremento de las hidrofitas (principalmente (yperaceae) en el centro de la RP y la expansión de la vegetación herbácea, mayormente representada por pastizales de gramíneas y una reducción de los taxones arbustivos en el sudoeste (Prieto, 2000). Durante la transición Pleistoceno-Holoceno (12.000-10.000 años cal. AP) se produjo el reemplazo de estepas secas por pastizales húmedos (Fig. 7) y una rápida evolución de ambientes lagunares que no fue sincrónico en la RP. Esta información está suplementada con datos polínicos provenientes de otros tipos de secuencias de la RP (Prieto, 1996, 2000).

Un aspecto destacable es la variabilidad que muestran las secuencias palinológicas continentales del Holoceno Medio y Tardío en contraste con las costeras. Las secuencias continentales, principalmente las ubicadas en el sudoeste de la RP (sitios 12, 13, 14, 15, Tab. 1), presentan fluctuaciones abruptas de los principales tipos polínicos (Poaceae, Asteraceae, Brassicaceae, Chenopodiaceae y Apiaceae) para cada registro, con varios niveles estratigráficos con evidencias de preservación diferencial o totalmente estériles, a excepción de las secuencias Arroyo Sauce Chico (sitio 17, Tab. 1), La Toma (sitio 11, Tab. 1) y Terraza II (sitio 12, Tab.1), estas dos últimas ubicadas en el Río Sauce Grande. Las secuencias ubicadas en el centro de la RP (sitios 3, 5, 6, Tab. 1) registran los cambios locales de la vegetación y las diferencias estarían relacionadas con la geomorfología y el tipo de sucesión sedimentaria analizada (ver caso de estudio).

Las secuencias ubicadas próximas a la costa atlántica muestran una gran continuidad espacial y reflejan procesos similares y homologables. En general, presentan espectros palinológicos con buena preservación, con excepción del 
sitio 16 (Tab. 1) que tiene numerosos niveles estériles (Quattrocchio et al., 1998) y el sitio 7 (Tab. 1) donde los palinomorfos entre ca. 3900 y $3000{ }^{14} \mathrm{C}$ años AP tienen mala preservación (Vilanova et al., 2006). Las diferencias espacio-temporales están relacionadas con la ubicación de cada secuencia con respecto a la línea de costa durante el Holo- ceno Medio y Tardío. Los cambios de nivel del mar influenciaron el desarrollo de las comunidades vegetales locales, y las secuencias palinológicas (sitios 2, 7, 8, 10, Tab. 1) registran la historia de la vegetación y de los ambientes en relación con la evolución geomorfológica de las llanuras costeras.

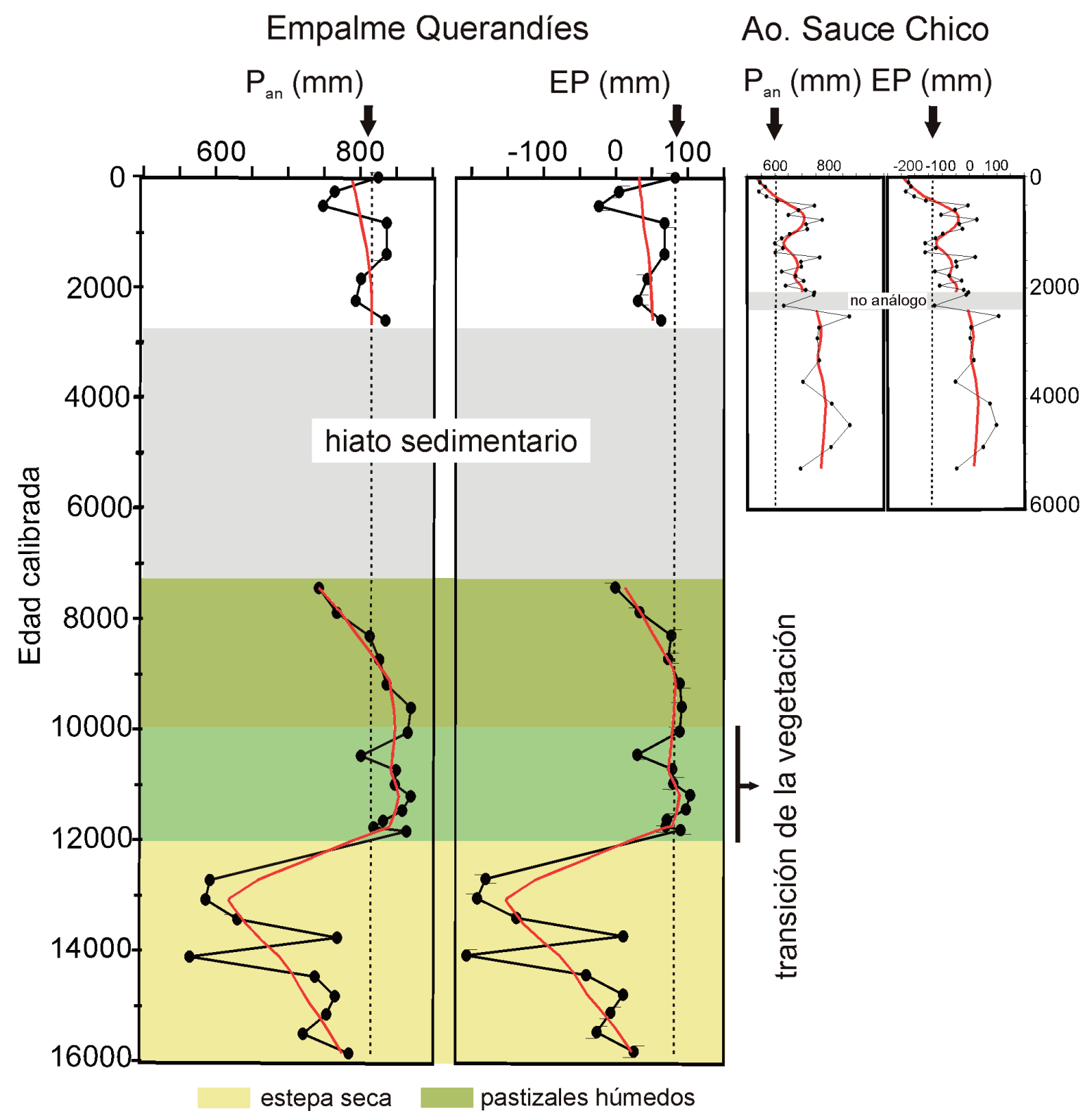

Figura 7. Cronología de las tendencias climáticas y cambios de la vegetación en la región pampeana. Curvas de los valores estimados de la precipitación anual (Pan) y la eficiencia de la precipitación (EP) a partir de las secuencias polínicas Empalme Querandíes (sitio 3, Fig. 1) y Arroyo Sauce Chico (sitio 17, Fig. 1). Las flechas y las líneas de puntos indican los valores actuales (modificado de Tonello y Prieto, 2010). 
La reconstrucción de la vegetación para el Holoceno Medio y Tardío realizada a partir del análisis polínico de sucesiones aluviales se ha complementado y ajustado con aquellos datos provenientes de otros tipos de secuencias, como las de lagos someros (e.g., Stutz et al., 2012, 2014) que han permitido reconstruir los principales cambios regionales de la vegetación. Las modificaciones de la vegetación durante el Holoceno Medio y Tardío estuvieron relacionadas con cambios en el nivel de base de los ríos y arroyos, la formación de estuarios en sus desembocaduras como consecuencia de las fluctuaciones del nivel del mar y los cambios de las condiciones climáticas durante ese tiempo.

Los trabajos discutidos han demostrado la importancia de estudiar varias secuencias que representan diferentes posiciones a lo largo de la llanura de inundación, con el fin de entender la dinámica de la vegetación y la evolución del ambiente durante el Holoceno dentro de una misma cuenca. La información regionalmente replicable y consistente derivada de secuencias palinológicas de varias cuencas de drenaje en el sureste de la RP, mostró que el análisis palinológico de los depósitos aluviales ha sido útil para reconstruir cambios paleoambientales en las áreas costeras y que son altamente sensibles a los cambios del clima y del nivel del mar.

Los datos polínicos de sucesiones aluviales de la RP (sitios 3, 13, Fig. 1) también han sido utilizados para la reconstrucción de la vegetación natural potencial de América Latina durante el Holoceno Medio (6000 $\pm 500{ }^{14} \mathrm{C}$ años AP), mediante el método de biomización (Marchant et al., 2009).

\section{Reconstrucción del clima}

El polen fósil de las sucesiones aluviales se ha utilizado como registro proxy indirecto del clima del pasado. Algunas de las inferencias climáticas para el final del Pleistoceno Tardío (anterior a 9900-10.440 ${ }^{14} \mathrm{C}$ años AP) se han basado en la ausencia de polen en las secuencias aluviales (e.g., Quattrocchio et al., 1998, 2008; Grill et al., 2007; Gutiérrez et al., 2011). Estas inferencias son controvertidas y la confiabilidad de las mismas ha sido cuestionada en la sección preservación polínica y tafonomía.

En numerosos trabajos, las inferencias climáticas para el Holoceno a partir de secuencias polínicas de sucesiones aluviales se realizaron utilizando la relación vegetaciónpolen-clima de manera cualitativa. Uno de los factores que ha dificultado el conocimiento del clima a partir de los registros palinológicos costeros es la influencia marina, cuyos efectos se solapan con los climáticos especialmente durante el tiempo en el que el nivel del mar fue más alto que el actual. Separar esos efectos no es sencillo, porque la mayor parte de las veces ambos interconectan sus causas y efectos. Por tal razón, la utilización de otros datos proxies en conjunto con el análisis palinológico en varias de estas secuencias ha facilitado la realización de inferencias climáticas (e.g., Vilanova y Prieto, 2012; Prieto et al., 2014).

La investigación sobre la relación cuantitativa polenclima en la RP se inició a principios del siglo XXI. Tonello y Prieto (2010) propusieron la aplicación de la técnica de análogos modernos a datos polínicos fósiles provenientes de dos sucesiones aluviales de la RP para obtener estimaciones cuantitativas de la precipitación y la eficiencia de la precipitación durante los últimos ca. 16.000 años cal AP (Fig. 7). Por otra parte, varias secuencias polínicas de sucesiones aluviales de la RP fueron incluidas como registros proxies para investigar la evolución temporal de las condiciones climáticas regionales del Holoceno Medio (6000 años cal. AP), combinando reconstrucciones a partir de múltiples indicadores y los modelos paleoclimáticos CMIP5/ PMIP3 (Coupled Model Intercomparison Project fifth phasel Paleoclimate Modeling Intercomparison Project 3) (Prado et al., 2013; Berman et al., 2016).

\section{CONSIDERACIONES FINALES}

La presente revisión ha mostrado que el análisis palinológico de sucesiones aluviales es un recurso importante de información paleoambiental para la RP, pero también ha expuesto que no todas las secuencias palinológicas publicadas son adecuadas para hacer reconstrucciones de la vegetación y del clima.

Se han documentado varios casos de depósitos aluviales con muestras polínicamente estériles o con polen comúnmente mal preservado, inclusive en algunos depósitos del Holoceno Tardío, indicando que un deterioro significativo del polen en estos depósitos puede ocurrir a escalas temporales breves. Aunque en este caso no brindarían información sobre la vegetación, la destrucción diferencial 
del polen es de interés para interpretar procesos tafonómicos, y por lo tanto debería tenerse en cuenta en los análisis paleoambientales. En este contexto, se recomienda no utilizar el término "presencia" de polen de uno o varios taxones para hacer interpretaciones de la vegetación y en cambio utilizarlo para indicar que algún proceso tafonómico ha alterado el espectro polínico (cf. Delcourt et al., 1980; Rojo et al., 2012), porque la "presencia" estaría sugiriendo que los escasos granos de polen que aparecen serían el resultado de la destrucción diferencial y/o mala preservación.

En algunos de los primeros trabajos publicados de la RP se interpretó la vegetación a partir de zonas polínicas con datos insuficientes (e.g., Grill, 1995; Borromei, 1998). Estas interpretaciones son inconsistentes y por lo tanto se recomienda no definir zonas polínicas cuando la mayoría de las muestras son estériles. Las zonas polínicas, entendidas como entidades temporales con composición polínica relativamente uniforme, se corresponden con las comunidades vegetales que son las unidades básicas de la clasificación en ecología vegetal (Seppä y Bennett, 2003) y es a partir de estas zonas polínicas que se interpreta la vegetación.

Las causas de la alteración de los conjuntos polínicos aluviales, aunque relacionadas principalmente con ciclos de hidratación-desecación, degradación microbiana y posible control de facies, han sido escasamente discutidas en la mayoría de los trabajos. Esto señala la necesidad de realizar estudios sobre la preservación polínica diferencial y la cuantificación del polen transportado por los ríos. Sería relevante poner más atención en el conocimiento de los procesos tafonómicos para mejorar la interpretación de los conjuntos polínicos fósiles de los depósitos aluviales. Documentar las clases de preservación polínica (e.g., Cushing, 1967; Delcourt y Delcourt, 1980) podría constituirse en una herramienta de utilidad al momento de analizar estos registros palinológicos para reconstrucciones ambientales. Por el contrario, existen numerosos depósitos aluviales del Holoceno donde la preservación del polen ha sido excelente y no se han observado distorsiones de los conjuntos polínicos debido al transporte fluvial, indicado por la información consistente y replicable derivada de varias secuencias palinológicas ubicadas en el sudeste de la RP.

Aún quedan amplias áreas con depósitos aluviales con excelentes afloramientos, que son potenciales para el análisis palinológico y permitirán ampliar la información regional sobre la vegetación del pasado. Es necesaria una exhaustiva búsqueda de secciones estratigráficas con probabilidades de obtener secuencias palinológicas bien preservadas, teniendo en cuenta que la experiencia ha demostrado que se pueden obtener en una misma sección estratigráfica secuencias con polen bien preservado y otras estériles. Sin dudas, el conocimiento de la geometría del cuerpo, de la variabilidad facial y de la historia geológica de los depósitos aluviales permitirá seleccionar varios perfiles estratigráficos a lo largo de una sucesión aluvial para los análisis palinológicos que brindarán una información espacio-temporal más adecuada y representativa y así, reconstruir la historia de la vegetación y realizar inferencias paleoambientales confiables.

\section{AGRADECIMIENTOS}

Deseo expresar mi agradecimiento a Mirta E. Quattrocchio por introducirme en el mundo de la Palinología y permitirme ser crítico, en muchas ocasiones incluso, con sus interpretaciones sobre palinología del Cuaternario. A M. Martínez y D. Olivera por invitarme a contribuir con un trabajo y por editar este volumen temático dedicado a Mirta. Este trabajo fue financiado con subsidios de la Universidad Nacional de Mar del Plata (Exa 695/14), el CONICET (PIP 543/14) y el FONCYT (PICT 950/14). A M. Zárate por las discusiones sobre la evolución de los depósitos aluviales de la región pampeana. A S. Stutz por sus comentarios y críticas y a los revisores por sus comentarios y sugerencias que contribuyeron a mejorar la primera versión de este trabajo. M.V. Mancini mejoró con sus comentarios y sugerencias la versión final.

\section{BIBLIOGRAFÍA}

Albert, B.M. y Pokorný, P. 2012. Pollen taphonomy and hydrology at Vranský potok versus Zahájí alluvial pollen sites: Methodological implications for cultural landscape reconstruction in the Peruc Sandstone Area, Czech Republic. Interdisciplinaria archaeological. Natural Sciences in Archaeology 3: 85-101.

Baker, R.G., Fredlund, G.G, Mandel, R.D. y Bettis III, E.A. 2000. Holocene environments of the central Great Plains: multi-proxy evidence from alluvial sequences, southeastern Nebraska. Quaternary International 67: 75-88.

Berman, A.L., Silvestri, G.E., Rojas, M. y Tonello, M.S. 2016. Accelerated greenhouse gases versus slow insolation forcing induced climate changes in southern South America since the MidHolocene. Climate Dynamics. DOI 10.1007/s00382-016-3081-z.

Bisernia, G. y van Geel, B. 2005. Reconstruction of Holocene palaeoenvironment and sedimentation history of the Ombrone alluvial plain (South Tuscany, Italy). Review of Palaeobotany and Palynology 136: 16-28.

Borel, C.M. 2007. Algas no silíceas y acritarcos de depósitos costeros holocenos en el Arroyo La Ballenera, Buenos Aires, Argentina. Ameghiniana 44: 359-366.

Borel, C.M., Bianchinotti, M.V. y Quattrocchio, M.E. 2001. Palinomofos fúngicos del Pleistoceno-Holoceno en el valle del Arroyo 
Chasicó, provincia de Buenos Aires, Argentina. Polen 11: 21-37.

Borromei, A.M. 1995. Palinología, estratigrafía y paleoambientes del Pleistoceno tardío-Holoceno en el valle del río Sauce Grande, provincia de Buenos Aires. Polen 7: 19-31.

Borromei, A.M. 1998. Vegetación y clima del Cuaternario tardío en el valle superior del Río Sauce Grande, Provincia de Buenos Aires, Argentina. Polen 9: 5-15.

Brown, A.G. 1985. The potential use of pollen in the identification of suspended sediment sources. Earth Surface Processes Landforms 10: 27-32.

Brown, A.G., Carpenter, R.G. y Walling, D.E. 2007. Monitoring fluvial pollen transport, its relationship to catchment vegetation and implications for palaeoenvironmental studies. Review of Palaeobotany and Palynology 147: 60-76.

Brown, A.G., Carpenter, R.G. y Walling, D.E. 2008. Monitoring the fluvial palynomorph load in a lowland temperate catchment and its relationship to suspended sediment and discharge. Hydrobiologia 607: 27-40.

Brush, G.S. y Brush, L.M. Jr. 1972. Transport of pollen in a sedimentladen channel, a laboratory study. American Journal of Science 272: 359-381.

Bryant, V.M. Jr. y Holloway, R.G. 1983. The role of palynology in archaeology. Advances in archaeological method and theory 6: 191-224.

Bryant, V.M. Jr., Holloway, R.G., Jones, J.G. y Carlson, D.L. 1994. Pollen preservation in alkaline soils of the American Southwest. En: A. Traverse (Ed.), Sedimentation of organic particles. Cambridge University Press, Cambridge, p. 47-58.

Bunting, M.J. y Tipping, R. 2000. Sorting dross from data: possible indicators of post depositational assemblage biasing in archaeological palynology. En: G. Bailey, N. Winder y R. Charles (Eds.), Human Ecodynamics. Oxbox Books, Oxford, p. 63-68.

Campbell, I.D. 1991. Experimental mechanical destruction of pollen grains. Palynology 15: 29-33.

Campbell, I.D. y Campbell, C. 1994. Pollen preservation: experimental wet-dry cycles in saline and desalinated sediments. $P a-$ lynology 18: 5-10.

Campbell, I.D. y Chmura, G.L. 1994. Pollen distribution in the Atchafalaya River, U.S.A. Palynology 18: 55-65.

Catto, N.R. 1985. Hydrodynamic distribution of palynomorphs in a fluvial succession, Yukon. Canadian Journal of Earth Science 22: 1552-1556.

Chmura, G.L. y Liu, K.B. 1990. Pollen in the lower Mississippi River. Review of Palaeobotany and Palynology 64: 253-261.

Chmura, G.L., Smirnov, A. y Campbell, I.D. 1999. Pollen transport through distributaries and depositional patterns in coastal waters. Palaeogeography, Palaeoclimatology, Palaeoecology 149: 257-270.

Cushing, E.J. 1967. Evidence for differential pollen preservation in Late Quaternary sediments in Minnesota. Review of Palaeobotany and Palynology 4: 87-101.

D'Antoni, H.L., Nieto, M.A. y Mancini, M.V. 1985. Pollen analytic stratigraphy of Arroyo Las Brusquitas profile (Buenos Aires Province, Argentina). Zentralblatt für Geologie und Paläontologie 1: 1721-1729.

Delcourt, P.A. y Delcourt, H.R. 1980. Pollen preservation and quaternary environmental history in the Southeastern United States. Palynology 4: 215-231.

Delcourt, P.A., Delcourt, H.R., .Brister, D.C. y Lackey, L.E. 1980. Quaternary vegetation history of the Mississippi embayment. Quaternary Research 13: 111-132.
Dimbleby, G.W. 1957. Pollen analysis of terrestrial soils. New Phytologist 56: 12-28.

Dimbleby, G.W. 1985. The palynology of archaeological sites. Academic Press, Inc., London, 176 p.

Elsik, W.C. 1971. Microbial degradation of sporopollenin. En: P. Brooks, R. Grant y M. Muir (Eds.), Sporopollenin. Proceeding of a Symposium at the Geological Department, Imperial College, London, p. 480-510.

Fall, P.L. 1987. Pollen taphonomy in a canyon stream. Quaternary Research 28: 393-406.

Fidalgo, F., De Francesco, F. y Colado, U. 1973. Geología superficial de las hojas Castelli, J.M. Cobo y Monasterio, Provincia de Buenos Aires. $5^{\circ}$ Congreso Geológico Argentino (Córdoba), Actas 4: 27-39.

Fucks, E.E., Schnack, E.J. y Aguirre, M.L. 2010. Nuevo ordenamiento estratigráfico de las secuencias marinas del sector continental de la bahía Samborombón, provincia de Buenos Aires. Revista de la Asociación Geológica Argentina 67: 27-39.

Grichuk, M.P. 1967. The study of pollen spectra from recent and ancient alluvium. Review of Palaeobotany and Palynology 4: $107-112$.

Grill, S.C. 1995. Análisis palinológico de un perfil cuaternario en la cuenca media del arroyo Napostá Grande, Localidad García del Río, provincia de Buenos Aires. $4^{\circ}$ Jornadas Geológicas Bonaerenses (Junín), Actas 1: 99-107.

Grill, S.C. 1997. Análisis palinológico de un perfil cuaternario en la cuenca media del arroyo Napostá Grande, provincia de Buenos Aires, Argentina. Implicancias paleoambientales. Polen 8: 23-40.

Grill, S.C. 2003. Análisis palinológico de sedimentos cuaternarios en la cuenca inferior del río Quequén Salado, provincia de Buenos Aires, Argentina. Polen 12: 37-52.

Grill, S., Borromei, A., Martínez, G., Gutiérrez, M.A., Cornou, M.E. y Olivera, D. 2007. Palynofacial analysis in alkaline soils and paleoenvironmental implications: The Paso Otero 5 archaeological site (Necochea district, Buenos Aires province, Argentina). Journal of South American Earth Sciences 24: 34-47.

Gutiérrez, M.A., Martínez, G., Luchsinger, H., Grill, S., Zucol, A.F., Hassan, G.S., Barros, M.P., Kaufmann, C.A. y Álvarez, M.C. 2011. Paleoenvironments in the Paso Otero locality during Late Pleistocene-Holocene (Pampean region, Argentina): An interdisciplinary approach. Quaternary International 245: 37-47.

Hall, S.A. 1977. Late Quaternary sedimentation and paleoecologic history of Chaco Canyon, New Mexico. Geological Society of American Bulletin 88: 1593-1618.

Hall, S.A. 1981. Deteriorated pollen grains and the interpretation of Quaternary pollen diagrams. Review of Palaeobotany and Palynology 32: 193-206.

Hall, S.A. 1985. Quaternary pollen analysis and vegetational history of the southwest. En: V.M. Bryant Jr. y R.G. Holloway (Eds.), Pollen Records of Late Quaternary North American Sediments. American Association of Stratigraphic Palynologist Foundation, Texas, p. 95-124.

Hall, S.A. 1989. Pollen analysis and paleoecology of alluvium. Quaternary Research 31: 435-438.

Hall, S.A. 1995. Late Cenozoic palynology in the South-central United States: cases of post-depositional pollen destruction. Palynology 19: 85-93.

Hall, S.A. 2010. New interpretations of alluvial and paleovegetation records from Chaco Canyon, New Mexico. En: J.E. Fassett, K.E. Zeigler y V.W Lueth (Eds.), Geology of the Four Corners Country. New Mexico Geological Society Guidebook, 61 ${ }^{\text {st }}$ Annual Fall 
Field Conference, p. 231-246.

Havinga, A.J. 1964. Investigation into the differential corrosion susceptibility of pollen and spores. Pollen et Spores 6: 621-635.

Havinga, A.J. 1967 Palynology and pollen preservation. Review of Palaeobotany and Palynology 2: 81-98.

Havinga, A.J. 1970. An experimental investigation into decay of pollen and spores in various soil types. En: P. Brooks, R. Grant y M. Muir (Eds.), Sporopollenin. Proceeding of a Symposium at the Geological Department, Imperial College, London, p. 446-479.

Havinga, A.J. 1984. A 20-year experimental investigation into the differential corrosion susceptibility of pollen and spores in various soil types. Pollen et Spores 26: 541-558.

Holloway, R.G. 1989. Experimental mechanical pollen degradation and its application to Quaternary age deposits. The Texas Journal of Science 41: 131-145.

Holmes, P.L. 1990. Differential transport of spores and pollen: A laboratory study. Review of Palaeobotany and Palynology 64: 289-296.

Horowitz, A. 1992. Palynology of arid lands. Elsevier, Amsterdam, $546 \mathrm{p}$.

Johnson, E., Holliday, V.T., Martínez, G., Gutiérrez, M. y Politis, G. 2012. Geochronology and landscape development along the middle Río Quequén Grande at the Paso Otero Locality, Pampa Interserrana, Argentina. Geoarchaeology: An International Journal 27: 300-323.

Ma, Y., Zhang, H., Pachur, H.J., Wünnemann, B., Li, J. y Feng, Z. 2004. Modern pollen-based interpretations of mid-Holocene palaeoclimate (8500 to $3000 \mathrm{cal}$. BP) at the southern margin of the Tengger Desert, northwestern China. The Holocene 14: 841-850.

Madrid, P. y Politis, G. 1991. Estudios paleoambientales en la región pampeana: un enfoque multidisciplinario: $9^{\circ}$ Congreso $\mathrm{Na}$ cional de Arqueología Chilena (Santiago de Chile), Actas 1: 131-154.

Marchant, R., Harrison, S.P., Hooghiemstra, H., Markgraf, V., van Boxel, J.H., Ager, T., Almeida, L., Anderson, R., Baied, C., Behling, H., Berrio, J.C., Burbridge, R., Björck, S., Byrne, R., Bush, M.B., Cleef, A.M., Duivenvoorden, J.F., Flenley, J.R., De Oliveira, P., van Geel, B., Graf, K.J., Gosling, W.D., Harbele, S., van der Hammen, T., Hansen, B.C.S., Horn, S.P., Islebe, G.A., Kuhry, P., Ledru, M.P., Mayle, F.E., Leyden, B.W., Lozano-García, S., Melief, A.B.M., Moreno, P., Moar, N.T., Prieto, A., van Reenen, G.B., Salgado-Labouriau, M.L., Schäbitz, F., Schreve-Brinkman, E.J. y Wille, M. 2009. Pollen-based biome reconstructions for Latin America at 0,6000 and 18,000 radiocarbon years. Climate of the Past 5: $725-767$

Martin, P.S. 1963. The last 10000 years: A fossil pollen record of the America Southwest. University of Arizona Press, Tucson, $87 \mathrm{p}$.

Mehringer, P.J. 1967. Pollen analysis and the alluvial chronology. Kiva 32: 96-101.

Mehringer, P.J., Martin, P.S. y Haynes, C.V. 1967. Murray Springs, a mid-postglacial pollen record from southern Arizona: American Journal of Science 265: 786-797.

Moss, P.T., Kershaw, A.P. y Grindrod, J. 2005. Pollen transport and deposition in riverine and marine environments within the humid tropics of northeastern Australia. Review of Palaeobotany and Palynology 134: 55-69.

Prado, L.F., Wainer, I., Chiessi, C.M., Ledru, M.-P. y Turcq, B. 2013. A mid-Holocene climate reconstruction for eastern South America. Climate of the Past 9: 2117-2133.

Prieto, A.R. 1989. [Palinología de Empalme Querandies (Provincia de Buenos Aires). Un modelo paleoambiental para el Pleistoceno tar-
dío-Holoceno. Tesis Doctoral, Facultad de Ciencias Exactas y Naturales, Universidad Nacional de Mar del Plata, Mar del Plata, 207 p. Inédita.].

Prieto, A.R. 1996. Late Quaternary vegetational and climatic changes in the Pampa grassland of Argentina. Quaternary Research 45: 73-88.

Prieto, A.R. 2000. Vegetational history of the Late glacial Holocene transition in the grasslands of eastern Argentina. Palaeogeography, Palaeoclimatology, Palaeoecology 157: 167188.

Prieto, A.R. y Quattrocchio, M.E. 1993. Briofitas y pteridofitas en sedimentos del Holoceno de la Provincia de Buenos Aires, Argentina. Anales de la Asociación de Palinólogos de Lengua Española 6: 17-37.

Prieto, A.R., Blasi, A.M., De Francesco, C.G. y Fernández, C. 2004. Environmental history since 11,000 yr B.P. of the northeastern Pampas, Argentina from alluvial sequences of Luján River. Quaternary Research 62: 146-161.

Prieto, A.R., Romero, M.V., Vilanova, I., Bettis III, E.A., Espinosa, M.A., Haj, A.E., Gómez, L. y Bruno, L.l. 2014. A multi-proxy study of Holocene environmental change from alluvial deposits, in the southern coast of the Pampa region, Argentina. Journal of Quaternary Science 29: 329-342.

Prieto, A.R., Vilanova, I. y De Francesco, C.G. 2003. Estratigrafía y paleoecología del Pleistoceno tardío-Holoceno del área del arroyo Las Brusquitas, Buenos Aires, Argentina: una revisión. En: M. Collantes, J.M. Sayago y L. del V. Neder (Eds.) $2^{\circ}$ Congreso Argentino de Cuaternario y Geomorfología, Cuaternario y Geomorfología (S.M. Tucumán), Actas: 47-56.

Prieto, A.R., Vilanova, I., Tonello, M.S. y Stutz, S. 2009. Reconstrucción de la vegetación y del clima de los pastizales pampeanos durante el Pleistoceno tardío-Holoceno a través del análisis palinológico. Quaternário do Rio Grande do Sul: integrando conhecimentos. Monografias da Sociedade Brasileira de Paleontologia: 107-120.

Quattrocchio, M.E., Borromei, A.M., Deschamps, C.M., Grill, S.C. y Zavala, C.A. 2008. Landscape evolution and climate changes in the Late Pleistocene-Holocene, southern Pampa (Argentina): Evidence from palynology, mammals and sedimentology. Quaternary International 181: 123-138.

Quattrocchio, M.E., Grill, S.C. y Zavala, C.A. 1998. Chronostratigraphic and Palynozone chronosequences charts of Napostá Grande Creek, Southwestern Buenos Aires Province, Argentina. Quaternary of South America and Antarctic Peninsula 7: 111-133.

Rabassa, J. 1989. Geología de los depósitos del Pleistoceno Superior y Holoceno en las cabeceras del río Sauce Grande, provincia de Buenos Aires. $1^{\circ}$ Jornadas Geológicas Bonaerense (1985, Tandil), Actas: 765-790.

Rojo, L.D., Mehl, A.E., Paez, M.M. y Zárate, M.A. 2012. Mid- to Late Holocene pollen and alluvial record of the arid Andean piedmont between $33^{\circ}$ and $34^{\circ} \mathrm{S}$, Mendoza, Argentina: Inferences about floodplain evolution. Journal of Arid Environments 77: $110-122$.

Seppä, H. 2013. Pollen Analysis, Principles. En: S.A. Elias (Ed.), Encyclopedia of Quaternary Science 3. Elsevier, Amsterdan, p. 794804.

Seppä, H. y Bennett, K.D. 2003. Quaternary pollen analysis: recent progress in palaeoecology and palaeoclimatology. Progress in Physical Geography 27: 548-579.

Smirnov, A., Chmura, G.L. y Lapointe, M.F. 1996. Spatial distribution of suspended pollen in the Mississippi River as an example of pollen transport in alluvial channels. Review of Palaeobotany 
and Palynology 92: 69-81.

Solomon, A.M., Blasing, T.J. y Solomon, J.A. 1982. Interpretation of floodplain pollen in alluvial sediments from an arid region. Quaternary Research 18: 52-71.

Stutz, S., Borel, C.M., Fontana, S.L. y Tonello, M.S. 2012. Holocene evolution of three shallow lakes in the SE Pampa plain (Argentina) as evidenced by analyses of pollen, non-pollen palynomorphs and plant macrofossils. The Holocene 22: 1215-1222.

Stutz, S. y Prieto, A.R. 2003. Modern pollen and vegetation relationships in Mar Chiquita coastal lagoon area, southeastern Pampa grasslands, Argentina. Review of Palaeobotany and Palynology 126: 183-195.

Stutz, S., Prieto, A.R. e Isla, F.I. 1999. Cambios de la vegetación durante el Holoceno en el SE bonaerense: análisis polínico del paleoestuario del arroyo La Ballenera. Publicación Especial de la Asociación Paleontológica Argentina, 6: 65-69.

Stutz, S., Tonello, M.S., González Sagrario, M.A., Navarro, D. y Fontana, S.L. 2014. Historia ambiental de los lagos someros de la Ilanura Pampeana (Argentina) desde el Holoceno medio: Inferencias paleoclimáticas. Latin American Journal of Sedimentology and Basin Analysis 21: 119-138.

Tipping, R. 2000. Pollen preservation analysis as a necessity in Holocene palynology. En: J.P. Huntley y S. Stallibrass (Eds.), Taphonomy and interpretation. Symposia of the Association of Environmental Archaeologist, 14. Oxbox Books, Oxford, p. 23-33.

Tonello, M. y Prieto, A.R. 2003. Relaciones cuantitativas polenclima actual para el pastizal pampeano: primeros resultados. Revista del Museo Argentino de Ciencias Naturales, Nueva Serie 5: 317-327.

Tonello, M.S y Prieto, A.R. 2008. Modern vegetation-pollen-climate relationships for the Pampa grasslands of Argentina. Journal of Biogeography 35: 926-938.

Tonello, M.S y Prieto, A.R. 2010. Tendencias climáticas para los pastizales pampeanos durante el Pleistoceno tardío-Holoceno: estimaciones cuantitativas basadas en secuencias polínicas fósiles. Ameghiniana 47: 501-514.

Tonello, M.S., Zárate, M.A. y Mancini, M.V. 2002. Trazas radicales ferrosas en una secuencia aluvial del río Quequén Grande (Buenos Aires): implicancias estratigráficas y ambientales. Ameghiniana 39: 163-174.

Traverse, A. 1992. Organic fluvial sediment: palynomorphs and "palynodebris" in the lower Trinity River, Texas. Annals of the Missouri Botanical Garden 79: 110-125.

Tschudy, R.H. 1969. Relationship of palynomorphs to sedimentation. En: R.H. Tschudy y R.A. Scott (Eds.), Aspects of Palynology. John Wiley \& Sons Inc, New York, p. 79-96.

Twiddle, C.L. y Bunting, M.J. 2010. Experimental investigations into the preservation of pollen grains: A pilot study of four pollen types. Review of Palaeobotany and Palynology 162: 621-630.

van Geel, B. 2001. Non-pollen palynomorphs: En: J.P. Smol, H.J.B. Birks y W.M. Last (Eds.), Tracking Environmental Change Using Lake Sediments. Volumen 3: Terrestrial, Algal and Siliceous Indicators. Kluwer Academic Publishers, Dordrecht, The Netherlands, p. 99-120.
Vilanova, I. y Prieto, A.R. 2012. Historia de la vegetación de las llanuras costeras de la Bahía Samborombón ( 35,5 S), Argentina, desde $7800{ }^{14} \mathrm{C}$ años. Ameghiniana 49: 303-318.

Vilanova, I., Prieto, A.R. y Espinosa, M.E. 2006. Paleoenvironmental evolution and sea-level fluctuations along the southeastern Pampa grasslands coast of Argentina during the Holocene. Journal of Quaternary Science 21: 227-242.

Vilanova, I., Prieto, A.R., Stutz, S. y Bettis III, A. 2010. Holocene vegetation changes along the southeastern coast of the Argentinean Pampa grasslands in relation to sea-level fluctuations and climatic variability: palynological analysis of alluvial sequences from Arroyo Claromecó. Palaeogeography, Palaeoclimatology, Palaeoecology 298: 210-223.

Work, P.T., Semkenb, H.A. y Baker, R.G. 2005. Pollen, plant macrofossils and microvertebrates from mid-Holocene alluvium in east-central lowa, USA: Comparative taphonomy and paleoecology. Palaeogeography, Palaeoclimatology, Palaeoecology 223: 204-221.

Xu, Q., Yang, X., Wu, Ch., Meng, L. y Wang, Z. 1996. Alluvial pollen on the North China Plain. Quaternary Research 46: 270-280.

Zavala, C. y Quattrocchio, M. 2001. Estratigrafía y evolución geológica del río Sauce Grande (Cuaternario), provincia de Buenos Aires, Argentina. Revista de la Asociación Geológica Argentina 56: 25-37.

Zárate, M.A. 2005. El Cenozoico tardío continental de la Provincia de Buenos Aires: En: R.E. de Barrio, P.O. Etcheverry, M.F. Caballé y E. Llambías (Eds.), Geología y Recursos Naturales de la Provincia de Buenos Aires. Relatorio del $16^{\circ}$ Congreso Geológico Argentino. Asociación Geológica Argentina, Buenos Aires, p. 139-158.

Zárate, M.A., Espinosa, M.A. y Ferrero, L. 1998. Paleoenvironmental inplications of a Holocene diatomite, Pampa Interserrana, Argentina. Quaternary of South America and Antarctic Peninsula 11: 135-152.

Zárate, M.A., Kemp, R.A., Espinosa, M.A. y Ferrero, L. 2000. Pedosedimentary and palaeoenvironmental significance of a Holocene alluvial sequence in the southern Pampas, Argentina. The Holocene 10: 481-488.

Doi: 10.5710/PEAPA.27.09.2016.114

Recibido: 29 de abril de 2016

Aceptado: 27 de septiembre de 2016 